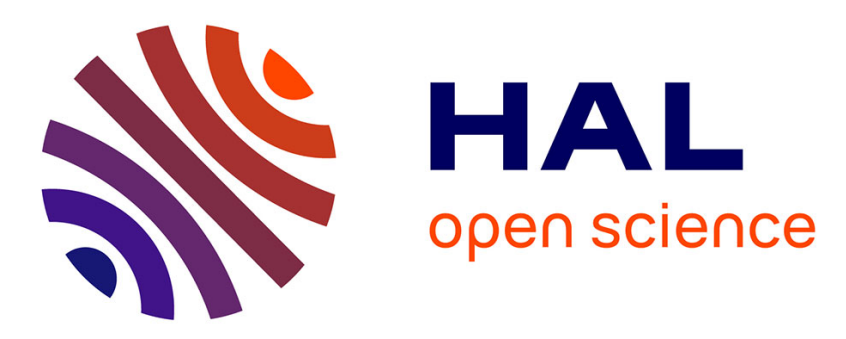

\title{
Copper-Catalyzed Anomeric O-Arylation of Carbohydrate Derivatives at Room Temperature
}

Tristan Verdelet, Sara Benmahdjoub, Belkacem Benmerad, Mouad Alami, Samir Messaoudi

\section{- To cite this version:}

Tristan Verdelet, Sara Benmahdjoub, Belkacem Benmerad, Mouad Alami, Samir Messaoudi. CopperCatalyzed Anomeric O-Arylation of Carbohydrate Derivatives at Room Temperature. Journal of Organic Chemistry, 2019, 84 (14), pp.9226-9238. 10.1021/acs.joc.9b01218 . hal-02396654

\section{HAL Id: hal-02396654 https://hal.science/hal-02396654}

Submitted on 6 Dec 2019

HAL is a multi-disciplinary open access archive for the deposit and dissemination of scientific research documents, whether they are published or not. The documents may come from teaching and research institutions in France or abroad, or from public or private research centers.
L'archive ouverte pluridisciplinaire HAL, est destinée au dépôt et à la diffusion de documents scientifiques de niveau recherche, publiés ou non, émanant des établissements d'enseignement et de recherche français ou étrangers, des laboratoires publics ou privés. 


\title{
Copper-catalyzed anomeric 0 -arylation of carbohydrate de- rivatives at room temperature
}

\author{
Tristan Verdelet, ${ }^{\mathrm{a}}$ Sara Benmahdjoub, ${ }^{\mathrm{b}}$ Belkacem Benmerad, ${ }^{\mathrm{b}}$ Mouad Alami, ${ }^{\mathrm{a},{ }^{*}}$ and Samir \\ Messaoudi $^{\mathrm{a}, *}$ \\ ${ }^{a}$ BioCIS, Univ. Paris-Sud, CNRS, University Paris-Saclay, Châtenay-Malabry, France \\ ${ }^{\mathrm{b}}$ Laboratoire de Physico-Chimie des Matériaux et Catalyse, Faculté des Sciences Exactes, Université de Bejaia, 06000 Be- \\ jaia, Algeria
}

Supporting Information Placeholder

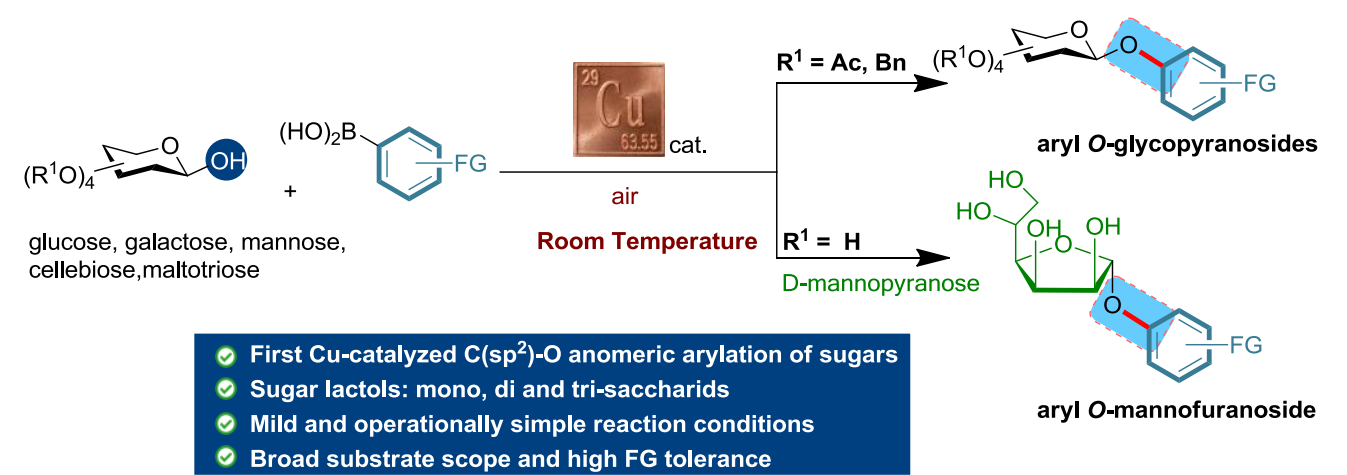

\begin{abstract}
Direct and practical anomeric $O$-arylation of sugars lactols with substituted aryl boronic acids has been established. Using copper catalysis at room temperature under air atmosphere, the protocol proved to be general, and a variety of aryl $O$-glycosides have been prepared in good to excellent yields. Furthermore, this approach was extended successfully to unprotected carbohydrates including $\alpha$-mannose, and demonstrated here how the interaction between carbohydrates and boronic acids can be combined with copper catalysis to achieve selective anomeric $O$-arylation.
\end{abstract}

\section{INTRODUCTION}

$O$-aryl glycosides are an important class of glycosides that exhibit promising biological activities, including antibiotic (e.g., vancomycin and chromomycin), antiviral, and anticancer (e.g., dauxorubucin) properties ${ }^{1}$ (Figure 1-A). Usually, these derivatives are prepared by treating phenol derivatives with glycosyl donors through an $S_{N^{2}}$ type mechanism under basic conditions or through an $\mathrm{S}_{\mathrm{N}} 1$ reaction under acidic conditions (Figure $1-B$, path 1 ). ${ }^{2}$ Glycosyl acetates, halides, and trichloroacetimidates have been used as donors in the formation of $\beta$-O-aryl glycosides, these later, however, only work well with specific phenol nucleophiles. For instance, glycosyl acetates and trichloroacetimidates are preferred for electron-donating phenol substrates. Glycosyl acetates usually provide the $\beta$-O-aryl glycosides with lower yields than trichloroacetimidates due to anomerization of both the glycosyl donor and the coupling product. ${ }^{2}$ Alternative routes to $O$-glycosidic bond formation consist on the use of phenols as partners in a Mitsunobu coupling glycosylation with sugar hemiacetal. ${ }^{3}$ However, the yield is clearly dependent on the acidity of the phenols, that is, the least acidic phenols give usually low yields. Nucleophilic aro- matic substitution $\left(\mathrm{S}_{\mathrm{N}} \mathrm{Ar}\right)$ reactions have been also used to generate $O$-arylglycosides from protected carbohydrates and activated fluoroarenes ${ }^{4}$ or electrophilic diaryliodonium salts ${ }^{5}$ (Figure 1-B, path 2). Other anomeric arylation methods that have been applied to hydroxyl group in protected sugars include photoredox substitution of aryl bromides under iridium catalysis ${ }^{6}$ (Figure 1-B, path 3). Although elegant, this method is restricted to aryl bromides bearing electron deficient groups. Besides, only per-O-benzylated sugar lactols were used as partners, thus limited the scope of the reaction. An 

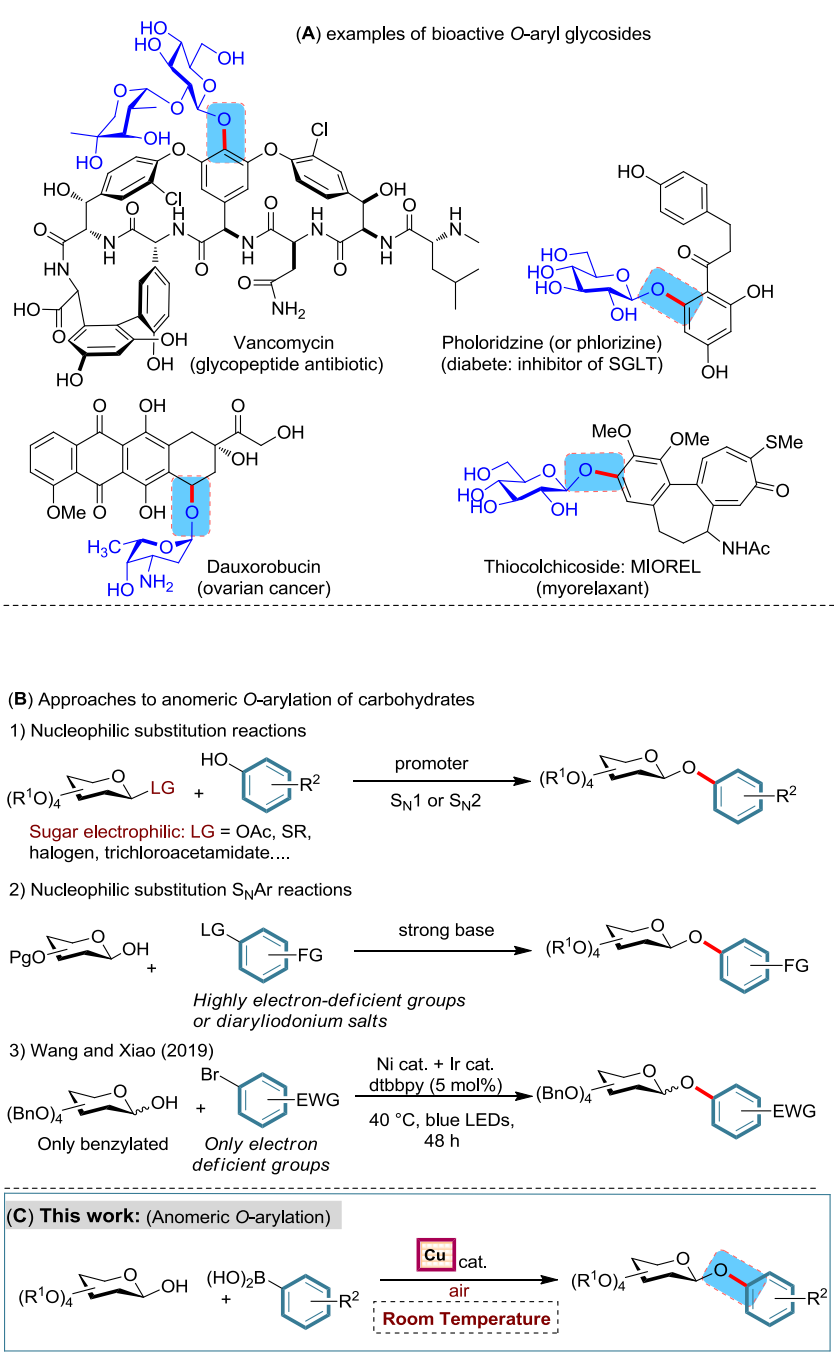

Figure 1. Example of bioactive $O$-aryl glycosides, and approaches to anomeric $O$-arylation of carbohydrates

appealing option to access aryl $O$-glycosides would be the use of carbohydrate hemiacetals as nucleophiles in transition metal-catalyzed reactions. Owing to the biological significance and existing restricted synthetic methodologies, there is an exigent need for a facile and efficient protocol to synthesize aryl $O$-glycosides. Since our initial reports on Chan-Lam-Evans cross coupling of $1^{-}$ aminosugars with arylboronic acids under copper catalysis, $^{7}$ to the best of our knowledge, there is no report describing the formation of aryl $O$-glycosides from carbohydrates hemiacetal and arylboronic acids. ${ }^{8}$ Consequently, in continuation of our interest in C-heteroatom bond formation, ${ }^{9}$ we report herein that $O$-arylation of sugar hemiacetals can be realized efficiently using a catalytic amount of $\mathrm{Cu}(\mathrm{OAc})_{2}$ at room temperature under air atmosphere (Figure $1-C$ ).

\section{RESULTS AND DISCUSSION}

In the first set of experiments, we examined the coupling of 2,3,4,6-tetra-O-acetyl-D-glucopyranose $\mathbf{1 a}$ (in a mixture of $\alpha / \beta=1: 3$ ) with phenylboronic acid $\mathbf{2 a}$ as a model study under various source of copper catalysts, bases and solvents. Representative results from this study are summarized in Table 1 . The reaction of $\mathbf{1 a}$ ( 1 equiv) with $\mathbf{2 a}$ (3 equiv) was first investigated under our previously reported procedure $\left[\mathrm{Cu}(\mathrm{OAc})_{2} \mathrm{H}_{2} \mathrm{O}\right.$ (20 mol\%), pyridine, $\mathrm{CH}_{2} \mathrm{Cl}_{2}, 24 \mathrm{~h}$ at $25^{\circ} \mathrm{C}$ ] (Table 1, entry 1). Unfortunately, this protocol afforded the desired aryl $\mathrm{O}$-glycoside $3 \mathrm{a}$ in a low $32 \%$ yield. Switching from $\mathrm{CH}_{2} \mathrm{Cl}_{2}$ as the solvent to the less volatile dichloroethane (DCE) furnished $3 \mathbf{a}$ in a slightly increased yield (39\%, entry 2). However in both cases, a significant erosion of the diastereomeric ratio $(\alpha / \beta$ ratio from 1:3 to 1:1.2) was observed. This result indicates clearly that the reactivity of hemiacetal carbohydrates is far to be similar to those of 1 -aminosugars and are more prone to anomerization than their aminocongeners. To increase the yield of $3 \mathbf{a}$ and reduce the erosion of the diastereomeric ratios, we examined the influence of other nitrogen bases under otherwise identical conditions. When DMAP, 2,6-lutidine or $\mathrm{Et}_{3} \mathrm{~N}$ were used instead pyridine, the yield has never exceed $15 \%$ demonstrating that pyridine is the best choice (compare entries 2 and 3-5). The optimization reaction conditions was continued with respect to copper catalyst, however, no significant improvement of the yield of 3 a was observed when $\mathrm{Cu}(\mathrm{acac})_{2}, \mathrm{Cu}(\mathrm{OTf})_{2}$ or $\mathrm{CuO}$ were used (entries 68). Extensive examinations of the other reaction parameters revealed that the use of molecular sieves plays a critical role in the outcome of the $\mathrm{C}\left(\mathrm{Sp}^{2}\right)-\mathrm{O}$ bond formation. Pleasingly, the yield of za was improved up to $73 \%$ with complete retention of the anomeric ratio $(\alpha / \beta=1: 3)$ when the

Table 1 Survey of reaction conditions for the $O$-arylation of Glu-(OAc) $)_{4}$ 1a with phenylboronic acid $\mathbf{2} \mathbf{a}^{\mathrm{a}}$

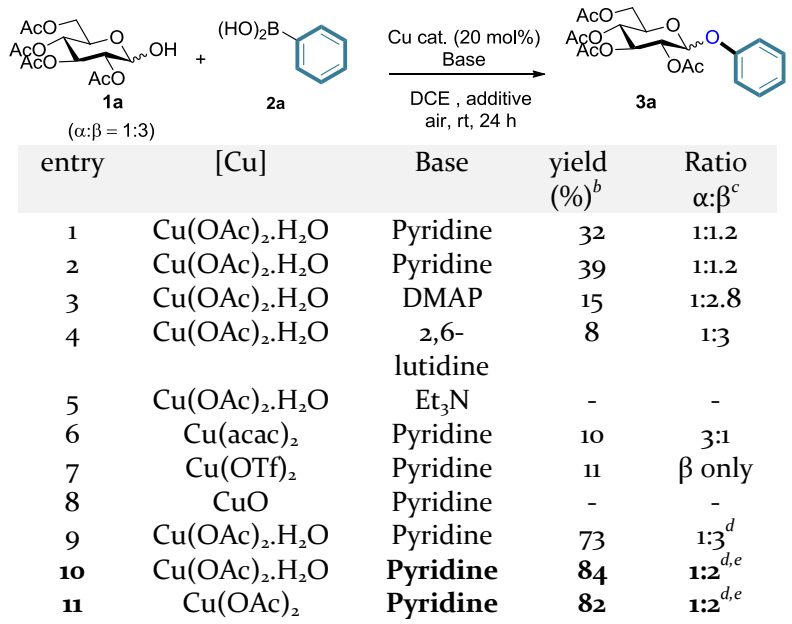

${ }^{a}$ 1a (1 equiv), phenylboronic acid 2a (3 equiv), [Cu] (20 mol \%), base (1 equiv), $24 \mathrm{~h}, \mathrm{rt},[0.5 \mathrm{M}] .{ }^{b}$ Yield of isolated 3a as a mixture of both anomers. ${ }^{c}$ anomer ratio was measured by ${ }^{1} \mathrm{H}$ NMR of the crude mixture and found to be the same when calculated after purification of both $\alpha: \beta$ anomers ${ }^{d}$ The reaction performed with molecular sieves as additive. ${ }^{e} 30$ $\mathrm{mol} \%$ of $\mathrm{Cu}(\mathrm{OAc})_{2} \cdot \mathrm{H}_{2} \mathrm{O}$ was used and reaction concentration increased to $[0.7 \mathrm{M}]$.

reaction was conducted in the presence of molecular sieves (entry 9). By increasing the amount of $\mathrm{Cu}$-catalyst (from $20 \mathrm{~mol} \%$ to $30 \mathrm{~mol} \%$ ) and the reaction concentration (from $0.5 \mathrm{M}$ to $0.7 \mathrm{M}$ ), we managed to isolate $84 \%$ of the desired product $3 \mathbf{a}$ with a slight erosion of the anomeric purity (from $\alpha: \beta$ 1:3 to 1:2) (entry 10). One can be noted that the use of anhydrous $\mathrm{Cu}(\mathrm{OAc})_{2}$ led to $3 \mathrm{a}$ in the same yield and same $\alpha / \beta$-ratio (1:2, entry 11). Importantly, the stability of 1a $(\alpha / \beta=1: 3)$ and both isolated pure anomeres $\alpha$-za and $\beta$-za in deuterated dichloromethane $\left(\mathrm{CD}_{2} \mathrm{Cl}_{2}\right)$ was measured by ${ }^{1} \mathrm{HNMR}$ after $19 \mathrm{~h}$ at room temperature. The results demonstrated that both anomeres $\alpha-3 \mathbf{a}$ and $\beta$-3a are completely stable in $\mathrm{CD}_{2} \mathrm{Cl}_{2}$ and 
no anomerization was detected by ${ }^{1} \mathrm{HNMR}$. However, analysis of the ${ }^{1} \mathrm{HNMR}$ of the starting material 1 a revealed that the anomeric purity was lost and the ratio $\alpha / \beta$ is 1:1.1. This result demonstrate cclearly that the erosion observed during the reaction is due to the anomerization of the starting glycosyl lactol (see SI, p98).

Motivated by these results, we next explored the scope of the coupling reaction of glucopyranose 1a (in a mixture of $\alpha / \beta=1: 3$ ) with various arylboronic acids. Gratifyingly, all the arylations proceeded cleanly to give the substituted aryl $O$-glycosides $\mathbf{3 a}-\mathbf{p}$ as a mixture of $\beta$ and $\alpha$ anomers in ratios ranging from $1: 2$ to $1: 7.3$ and yields up to $86 \%$. As Scheme 1 Scope of Arylboronic Acids 2 for $\mathrm{Cu}$-Catalyzed $O$-Arylation of $\mathbf{1 a}^{\text {a }}$
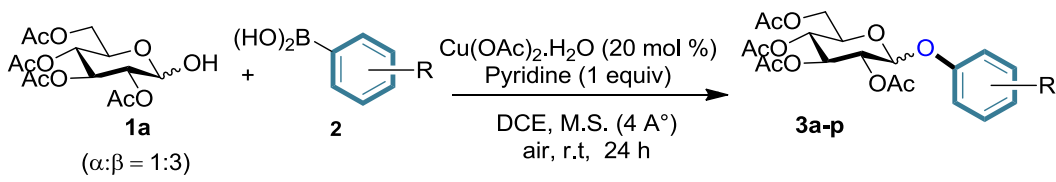

phenyl boronic acids
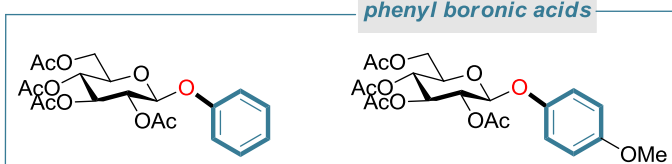

3a, $84 \%(\alpha / \beta=1: 2)^{*}$

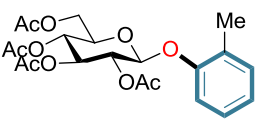

3e, traces
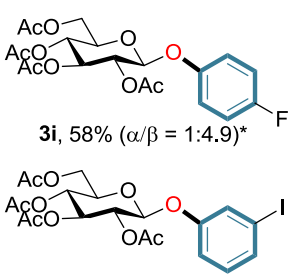

$3 m, 79 \%(\alpha / \beta=1: 3)^{\star}$ 3b, $59 \%(\alpha / \beta=1: 2.1)^{*}$

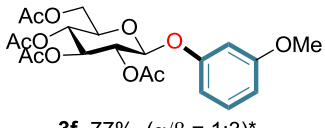

3f, $77 \%(\alpha / \beta=1: 3)^{*}$
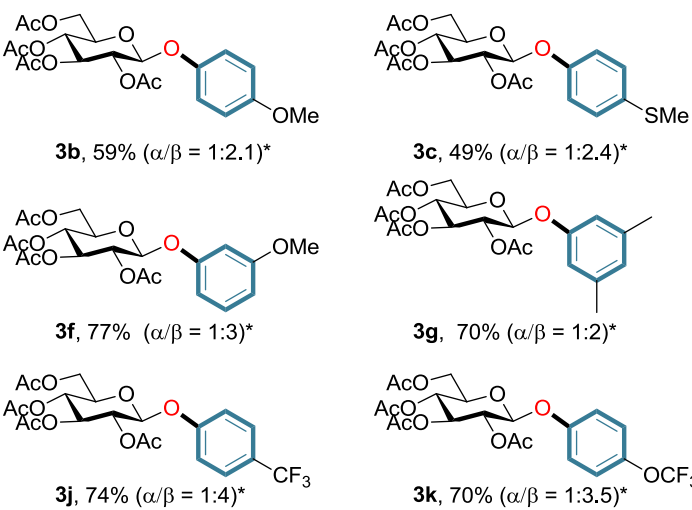

3c, $49 \%(\alpha / \beta=1: 2.4)^{\star}$

$$
\mathrm{AcO} 7
$$

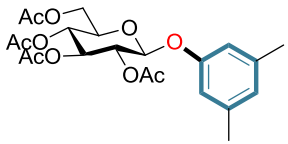

3g, $70 \%(\alpha / \beta=1: 2)^{\star}$
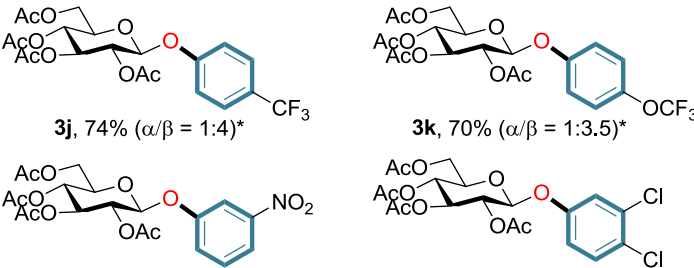

3n, $59 \%(\alpha / \beta=1: 4)^{*}$

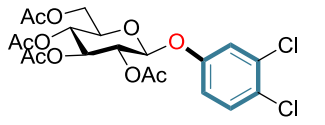

3o, $24 \%(\alpha / \beta=1: 7.3)^{\star}$

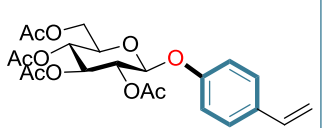

3d, $84 \%(\alpha / \beta=1: 2)^{*}$

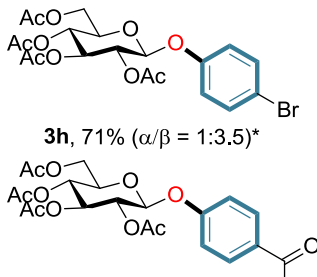

3I, $74 \%(\alpha / \beta=1: 3)^{*}$

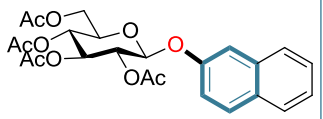

$3 p, 86 \%(\alpha / \beta=1: 2.2)$

Reaction conditions: reaction of 1 a (1 equiv) with $\operatorname{ArB}(\mathrm{OH})_{2} 2$ (3 equiv) were performed in flask at r.t. in DCE by using $\mathrm{Cu}(\mathrm{OAc})_{2} \mathrm{H}_{2} \mathrm{O}$ (30 mol \%), Pyridine (1 equiv) and molecular sieves. *anomer ratio was calculated after purification and isolation of both $\alpha$ - and $\beta$-products from the crude mixture. ${ }^{* *}$ anomer ratio was measured by ${ }^{1} \mathrm{H}$ NMR (and confirmed by HPLC) on the mixture of both anomers.

by LCMS analysis even when a stoichiometric amount of copper catalyst was used.

In a further set of experiments, we investigated the scope and generality of the method with respect to mono- diand polysaccharides. As depicted in Scheme 2, this coupling reaction tolerates a large variety of sugars $\mathbf{1 b}-\mathbf{h}$ and furnished the desired aryl $O$-glycosides in yields up to 90\%. At first we investigated the influence of the stereochemistry of the anomeric hydroxyl group on the coupling reaction. Thus, when the $\alpha / \beta$-ratio of tetra-acetoxyD-glucopyranose was inversed from 1:3 to 3:1 in favour of the $\alpha$-anomer $\mathbf{1 b}$, the coupling reaction with $\mathbf{2 a}$ proceeded smoothly giving the desired product $4 \mathbf{a}$ in $82 \%$ yield and
2:1 of anomeric purity. Moving from D-glucopyranose to $O$-acetylated $\alpha$-D-mannose ic $(\alpha / \beta=9: 1)$, and $O$ acetylated D-galactopyranose $\mathbf{1 d}(\alpha / \beta=1: 2.3)$ resulted in the isolation of compound $\mathbf{4 b}$ and $\mathbf{4 c}$ in excellent yields and

a slight loss of anomeric purity $(<5 \%)$. The synthetic potential of this protocol was demonstrated by performing a gram scale process with $1 \mathrm{c}(5.7 \mathrm{mmol})$. Delightedly, the reaction proceeded well to afford phenolic glycoside $\mathbf{4} \mathbf{b}$ in $94 \%$ yield with the anomeric purity $\alpha / \beta=6.1: 1$. Interestingly, joining of the phenylboronic acid moiety to $\alpha-\mathrm{D}$ mannofuranose 1e was

Scheme 2 Scope of Sugar Lactols $\mathbf{1 b}$-j for Cu-Catalyzed $O$-Arylation with phenylboronic Acids $\mathbf{2 a}{ }^{\text {a }}$

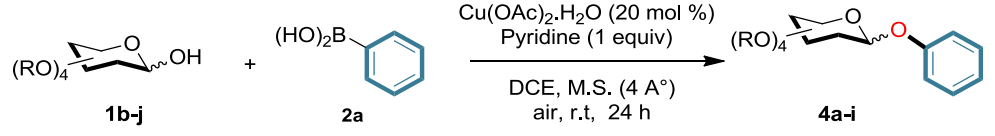




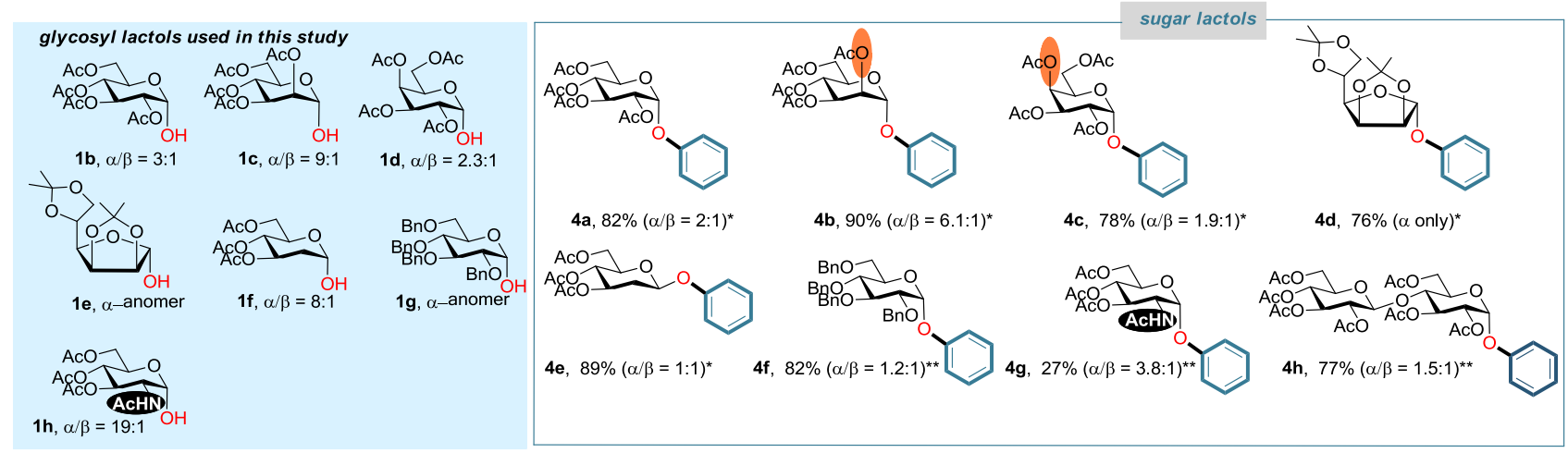

Reaction conditions: reaction of $\mathbf{1 b}-\mathbf{j}$ (1 equiv) with $\mathrm{PhB}(\mathrm{OH})_{2} \mathbf{2 a}$ (3 equiv) were performed in flask at r.t. in DCE by using $\mathrm{Cu}(\mathrm{OAc})_{2} \mathrm{H}_{2} \mathrm{O}$ (30 mol \%), Pyridine (1 equiv) and molecular sieves. *anomer ratio was calculated after purification and isolation of both $\alpha$ - and $\beta$-products from the crude mixture. ${ }^{*}$ anomer ratio was measured by ${ }^{1} \mathrm{H}$ NMR (and confirmed by HPLC) on the mixture of both anomers.

effective and resulted in the formation of the desired product $4 \mathrm{~d}$ in $76 \%$ yield as the only $\alpha$-anomer.

Next, the scope of the new reaction was investigated by using two glycosyl partners devoid from the C-2 ester neighboring participating group: the 2-deoxy glucose if and $\mathbf{1 g}$ bearing a benzyl group rather than the acetyl. As we can expect, when these two substrates where used, the coupling products $\mathbf{4} \mathbf{e}$ and $\mathbf{4} \mathbf{f}$ were isolated nicely in excellent $89 \%$ and $82 \%$ yields, respectively but the anomeric purity was completely lost in these cases. These results indicate that the acetyl group at the $\mathrm{C}_{2}$-position plays a pivotal role in maintaining the stability of the anomeric purity of glycosyl lactols if and $\mathbf{1 g}$ under the reaction conditions.

Scheme 3 Scope of the Cu-Catalyzed $O$-Arylation with more complex carbohydrates ${ }^{\text {a }}$

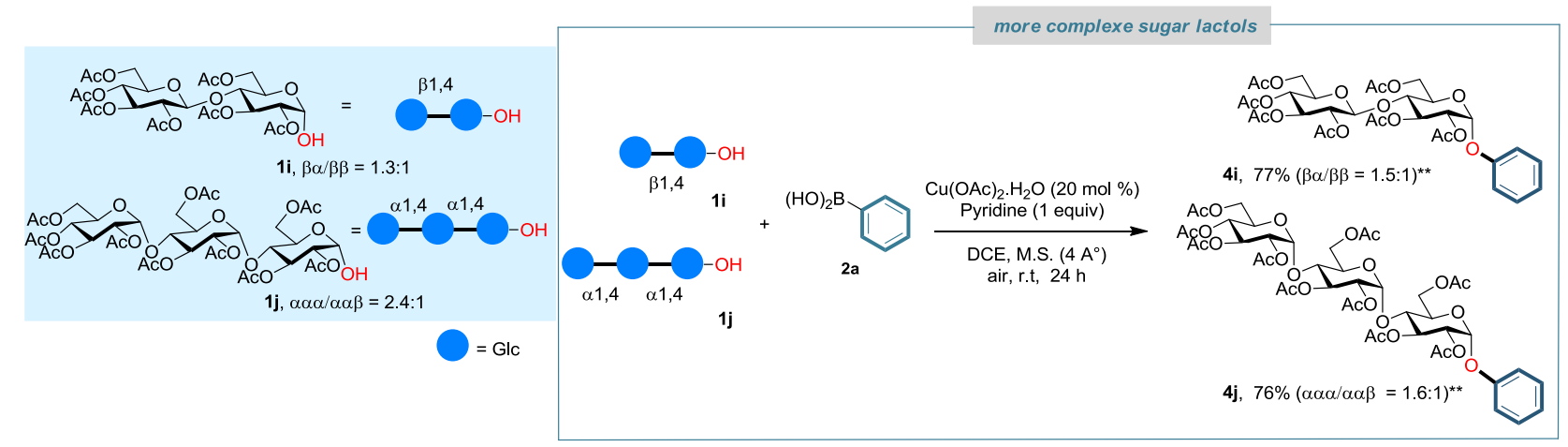

Reaction conditions: reaction of $\mathbf{1 i - j}$ (1 equiv) with $\mathrm{PhB}(\mathrm{OH})_{2}$ 2a (3 equiv) were performed in flask at r.t. in DCE by using $\mathrm{Cu}(\mathrm{OAc})_{2} \mathrm{H}_{2} \mathrm{O}$ (30 mol \%), Pyridine (1 equiv) and molecular sieves. ${ }^{* *}$ anomer ratio was measured by ${ }^{1} \mathrm{H}$ NMR (and confirmed by HPLC) on the mixture of both anomers.

Having demonstrated the efficacy of our method with various sugar lactols, we next turned our attention to the validation of the method with respect to more complex and biologically relevant saccharides (Scheme 3). We were delighted to find that di- and trisaccharide derivatives cellobiose $\mathbf{1 i}(\alpha / \beta=1.2: 1)$ and maltotriose $\mathbf{1 j}(\alpha / \beta=$ 3:1), readily undergo $O$-arylation coupling in this transformation. The desired $O$-aryl glycoside $\mathbf{4} \mathbf{i}$ was isolated in $77 \%(\alpha / \beta=1.1: 1)$. Moreover, the $O$-aryl trisaccharide $4 \mathbf{j}$ which is commonly used in bacterial imaging ${ }^{10}$ was isolated in $76 \%$ yield $(\alpha / \beta=1.6: 1)$. Importantly, the stereochemistry of the 1,4' -O-glycosidic bond in the disaccharides $\mathbf{4} \mathbf{i}$ and the $\alpha-1,4^{\prime}$ in trisaccharide $4 \mathbf{j}$ remained intact.

Of note in almost all cases, the $\beta$ and $\alpha$ anomers were separated by $\mathrm{SiO}_{2}$ flash chromatography or preparative HPLC and the NMR of pure single anomers has been reported (see SI).
It was well known that arylboronic acids react with nonprotected sugars having erythro-configured $\mathrm{OH}$ groups at $\mathrm{C}-2$ and $\mathrm{C}_{-3}$ (e.g., mannose, allose ${ }^{\mathrm{II}}$ to favor the formation of furanosides, with which they form particularly stable chelated complexes. Thus we were interesting whether this strategy may be applied to unprotected sugars to perform a selective $O$-arylation reaction of the anomeric $\mathrm{OH}$ group. In this context, we were pleased to observe that when the commercially available $\alpha-\mathrm{D}$ mannose $\mathbf{~ k ~}(\alpha / \beta=4: 1)$ and phenylboronic acid 2a (5 equiv) were subjected to our conditions, phenyl $O$ - furanoside 5a was generated in moderate yield as a single $\alpha$-anomer (Scheme 4 ). After reaction with $m$-CPBA to cleave boronic ester groups, $\mathbf{6 a}$ could be isolated in a good $80 \%$ yield despite the fact that the conditions were not been optimized.

Based on previous works ${ }^{12}$ on the mechanistic study of the Chan-Lam-Evans reaction, we proposed in Figure 2 a mechanistic description of the $\mathrm{O}$-arylation of sugar lactols. At first, the complex (I) is formed through 
denucleation of the complex $\left[\mathrm{Cu}(\mathrm{OAc})_{2}\right]_{2} \cdot 2 \mathrm{H}_{2} \mathrm{O}$. Engagement of the arylboronic acid leads to transmetalation via 4-membered transition state (II) to

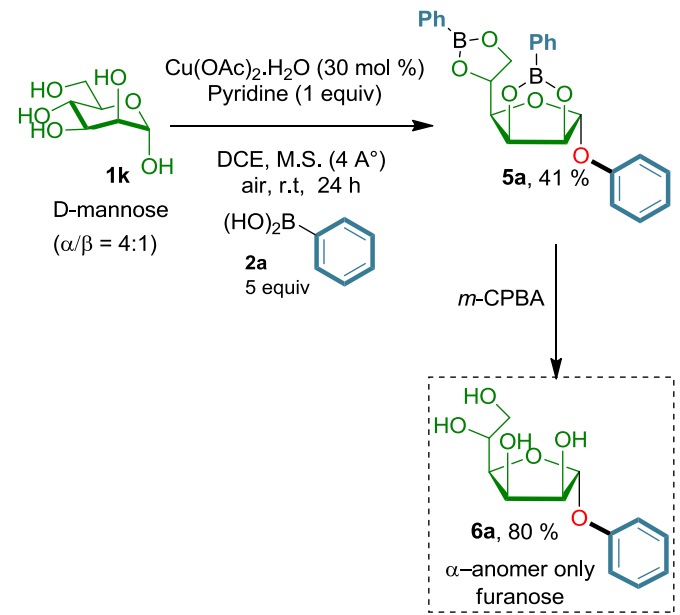

Scheme $4 \mathrm{Cu}$-catalyzed $\mathrm{O}$-arylation of unprotected $\alpha$-Man $\mathbf{1 k}$ with phenylboronic acid $\mathbf{2} \mathbf{a}^{\mathrm{a}}$

deliver $\mathrm{Cu}$ (II) species (III). Oxidation to $\mathrm{Cu}(\mathrm{III})$ via disproportionation of $\mathrm{Cu}^{\mathrm{II}}(\mathrm{OAc})_{2}$ to $\mathrm{Cu}^{\mathrm{I}}(\mathrm{OAc})$ gives complex (IV). A selective $\mathrm{C}-\mathrm{O}$ reductive elimination liberates the desired $\mathrm{O}$-arylglycoside and a $\mathrm{Cu}(\mathrm{I}) \mathrm{OAc}$ species (V). Completion of the catalytic cycle is achieved via oxidation to $\mathrm{Cu}$ (II) in the presence of $\mathrm{O}_{2}$ and $\mathrm{AcOH}$.

\section{CONCLUSION}

In summary, we have succeeded in achieving the coupling of sugar lactols with functionalized arylboronic acid at room temperature to furnish aryl $O$-arylglycosides. To the best of our knowledge the $\mathrm{C}\left(\mathrm{sp}^{2}\right)-\mathrm{O}$ bond of aryl $\mathrm{O}$ glycosides was formed, for the first time, directly by using anomeric hydroxyl group of sugars as nucleophile in the presence of $\mathrm{Cu}(\mathrm{OAc})_{2}$ as the catalyst system. Because of the mildness of the reaction conditions, the protocol developed is stereoretentive, functional-group tolerant, and proceeds in good to excellent yields. Given its distinct convenience we expect this method to be widely adopted within the synthetic and medicinal chemistry community.

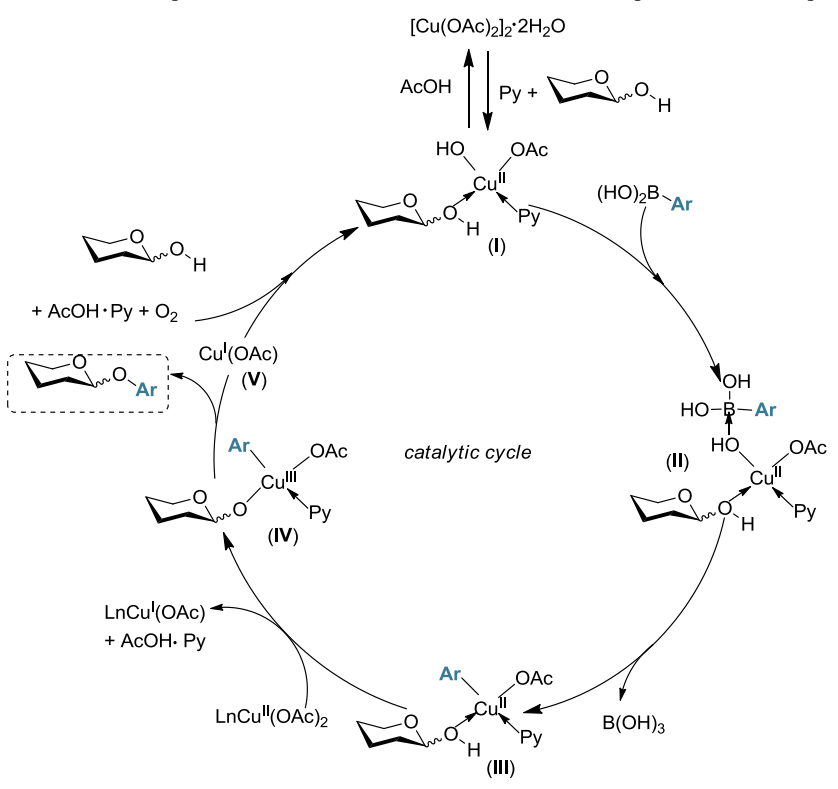

Figure 2. A proposed mechanism to anomeric $O$-arylation of carbohydrates

\section{EXPERIMENTAL SECTION}

General Procedure. Unknown compounds were all identified by usual physical methods, e.g., ${ }^{1} \mathrm{H} N M R,{ }^{13} \mathrm{C}$ NMR, IR, MS (ESI). ${ }^{1} \mathrm{H}$ and ${ }^{13} \mathrm{C}$ NMR spectra were measured in deuterated solvents with a Bruker Avance-30o. ${ }^{1} \mathrm{H}$ chemical shifts are reported in ppm from an internal standard TMS or of residual solvent peak. ${ }^{13} \mathrm{C}$ chemical shifts are reported in ppm from the residual solvent peak. IR spectra were measured on a Bruker Vector 22 spectrophotometer. Analytical TLC was performed on Merck precoated silica gel 6oF plates. Merck silica gel 6o (o.015$0.040 \mathrm{~mm}$ ) was used for column chromatography. High pressure liquid chromatography was recorded with a Waters Alliance 2695 device using a DAD 2996 as UV detector. High resolution mass spectra (HR-MS) were recorded on a Bruker MicroTOF spectrometer, using ESI with methanol as the carrier solvent. Nominal and exact $\mathrm{m} / \mathrm{z}$ values are reported in Daltons. 2,3,4,6-Tetra-Oacetyl- $\beta$-D-glucopyranose 1a $(\alpha: \beta=1: 3), \quad \beta$-D-glucose pentacetate, $\beta$-D-galactose pentacetate, D-mannose, $D$ glucosamine, 2,3,4,6-Tetra-O-benzyl- $\alpha$-D-glucopyranose 1g, maltotriose, $\alpha$-D-cellobiose octaacetate, tri-O-acetylD-glucal, 2,3,5,6-di-O-isopropylidene- $\alpha-\mathrm{D}$ mannofuranose 1e and all boronic acids were bought from available commercial sources.

\section{General procedure for copper-catalyzed anomeric $O$ - arylation of carbohydrate derivatives.}

In a $50 \mathrm{~mL}$ round bottom flask, monohydrated copper (II) acetate (30 $\mathrm{mg}, 0.15 \mathrm{mmol}$ ) and pyridine (40 $\mu \mathrm{L}, 0.50 \mathrm{mmol})$ were added on activated $4 \AA$ molecular sieves (200 mg). Dichloroethane $(700 \mu \mathrm{L}, \mathrm{c}=0.7 \mathrm{M})$ was added and the resulting suspension was stirred for 5 minutes. The boronic acid $(1.50 \mathrm{mmol})$ was then introduced and the reaction media was stirred again for 5 minutes. Finally, the carbohydrate $(0.50 \mathrm{mmol})$ was added and the resulting mixture was stirred at room temperature and under air atmosphere for 24 hours (the round bottom flask was capped to prevent the evaporation of dichloromethane). The media was then diluted with dichloromethane and filtered over celite. Purification by silica gel chromatography (cyclohexane/EtOAc) afforded the desired compound. Unless other specified indications, $\alpha$ and $\beta$ anomers were isolated separately.

\section{Description of coupling products $3 a-d, 3^{f}-p$ and $4 a-i$}

1-Phenyl-2,3,4,6-tetra-O-acetyl-D-glucopyranoside za.

The title compound was prepared according to the general procedure from commercially available 2,3,4,6-tetra-O-acetyl-D-glucopyranose (174 mg, 0.5 mmol, $\alpha: \beta=1: 3$ ) and phenylboronic acid (183 $\mathrm{mg}$ ) and was purified on silica gel chromatography (cyclohexane/EtOAc, from 9o/10 to 70/30) to afford $59 \mathrm{mg}$ of the $\alpha$ 
anomer and $115 \mathrm{mg}$ of the $\beta$ anomer as amorphous white solids. Global yield: $84 \%$ (o.42 mmol, $178 \mathrm{mg}$ ).

\section{1-Phenyl-2,3,4,6-tetra-O-acetyl- $\alpha$-D-glucopyranoside. ${ }^{13}$}

${ }^{1} \mathrm{H}$ NMR (300 $\left.\mathrm{MHz}, \mathrm{CDCl}_{3}\right) \delta$ 7.35-7.28 (m, $2 \mathrm{H}$ ), 7.12-7.05 $(\mathrm{m}, 3 \mathrm{H}), 5.75(\mathrm{~d}, J=3.3 \mathrm{~Hz}, 1 \mathrm{H}), 5.72($ app t, $J=9.8 \mathrm{~Hz}, 1$ H), 5.17 (app t, $J=9.8 \mathrm{~Hz}, 1 \mathrm{H}), 5.06(\mathrm{dd}, J=10.2,3.7 \mathrm{~Hz}, 1$ H), 4.27 (dd, $J=12.2,4.5 \mathrm{~Hz}, 1 \mathrm{H}$ ), 4.15 (ddd, $J=10.2,4.5$, $2.1 \mathrm{~Hz}, 1 \mathrm{H}), 4.07(\mathrm{dd}, J=12.2,2.2 \mathrm{~Hz}, 1 \mathrm{H}), 2.08(\mathrm{~s}, 3 \mathrm{H})$, $2.07(\mathrm{~s}, 3 \mathrm{H}), 2.06$ (s, $3 \mathrm{H}), 2.05(\mathrm{~s}, 3 \mathrm{H}) .{ }^{13} \mathrm{C}$ NMR $(75 \mathrm{MHz}$, $\left.\mathrm{CDCl}_{3}\right) \delta 170.6,170.2,169.6,156.1,129.7$ (2 C), 123.0, 116.6 (2 C), 94.2, 70.5, 70.1, 68.3, 68.0, 61.6, $20.6(4 \mathrm{C})$.

\section{1-Phenyl 2,3,4,6-tetra-O-acetyl- $\beta$-D-glucopyranoside. ${ }^{14}$}

${ }^{1} \mathrm{H}$ NMR (300 $\mathrm{MHz}, \mathrm{CDCl}_{3}$ ) $\delta$ 7.34-7.28 (m, $2 \mathrm{H}$ ), 7.11-7.07 (m, 1 H), 7.02-6.99 (m, $2 \mathrm{H})$, 5.35-5.26 (m, $2 \mathrm{H})$, 5.22-5.17 $(\mathrm{m}, 1 \mathrm{H}), 5.12-5.09(\mathrm{~m}, 1 \mathrm{H}), 4.31(\mathrm{dd}, J=12.2,5.3 \mathrm{~Hz}, 1 \mathrm{H})$, 4.18 (dd, $J=12.3,2.5 \mathrm{~Hz}, 1 \mathrm{H}$ ), 3.88 (ddd, $J=9.9,5.3,2.5$ Hz, $1 \mathrm{H})$, $2.09(\mathrm{~s}, 3 \mathrm{H}), 2.08(\mathrm{~s}, 3 \mathrm{H}), 2.06(\mathrm{~s}, 3 \mathrm{H}), 2.05$ (s, 3 H). ${ }^{13} \mathrm{C}$ NMR $\left(75 \mathrm{MHz}, \mathrm{CDCl}_{3}\right) \delta 170.6,170.2,169.4,169.3$, 156.8, 129.6 (2 C), 123.4, 117.0 (2 C), 99.1, 72.7, 72.0, 71.2, 68.3, 62.0, 20.7, $20.6(3 \mathrm{C})$.

\section{1-(4'-Methoxy)-2,3,4,6-tetra-O-acetyl-D- glucopyranoside 3 b.}

The title compound was prepared according to the general procedure from commercially available 2,3,4,6-tetraO-acetyl-D-glucopyranose (174 mg, $0.5 \mathrm{mmol}, \alpha: \beta=1: 3$ ) and 4-methoxyphenylboronic acid $(228 \mathrm{mg}$ ) and was purified on silica gel chromatography (cyclohexane/EtOAc, from 9o/10 to 70/30) to afford $43 \mathrm{mg}$ of the $\alpha$ anomer and $90 \mathrm{mg}$ of the $\beta$ anomer as amorphous white solids. Global yield: $59 \%$ (o.29 mmol, $134 \mathrm{mg}$ ).

\section{1-(4'-Methoxy)-2,3,4,6-tetra-O-acetyl- $\alpha$ - $D$ - glucopyranoside. ${ }^{15}$}

${ }^{1} \mathrm{H}$ NMR (300 MHz, $\mathrm{CDCl}_{3}$ ) $\delta$ 7.04-7.01 (m, $\left.2 \mathrm{H}\right), 6.86-6.83$ $(\mathrm{m}, 2 \mathrm{H}), 5.70$ (app t, $J=9.8 \mathrm{~Hz}, 1 \mathrm{H}), 5.63(\mathrm{~d}, J=3.7 \mathrm{~Hz}, 1$ H), $5.16($ app t, $J=9.8 \mathrm{~Hz}, 1 \mathrm{H}), 5.03(\mathrm{dd}, J=10.3,3.7 \mathrm{~Hz}, 1$ H), 4.27 (dd, $J=12.1,4.6 \mathrm{~Hz}, 1 \mathrm{H}$ ), 4.18 (ddd, $J=12.0,4.7$, $2.1 \mathrm{~Hz}, 1 \mathrm{H})$, 4.09 (dd, $J=12.1,2.1 \mathrm{~Hz}, 1 \mathrm{H}), 3.79(\mathrm{~s}, 3 \mathrm{H})$, 2.09-2.06 (m, 12 H). ${ }^{13} \mathrm{C}$ NMR (75 MHz, $\left.\mathrm{CDCl}_{3}\right) \delta$ 170.5, 170.1 (2 C), 169.6, 155.5, 150.2, 117.9 (2 C), 114.7 (2 C), 95.1, 70.6, 70.1, 68.4, 67.9, 61.7, 55.7, 20.7, 20.6 (3 C).

\section{1-(4'-Methoxy)-2,3,4,6-tetra-O-acetyl- $\beta$ - $D$ -} glucopyranoside. ${ }^{16}$

${ }^{1} \mathrm{H}$ NMR (300 $\mathrm{MHz}, \mathrm{CDCl}_{3}$ ) $\delta$ 6.98-6.95 (m, $2 \mathrm{H}$ ), 6.85-6.82 (m, $2 \mathrm{H}), 5.93-5.22(\mathrm{~m}, 1 \mathrm{H}), 5.17(\mathrm{t}, J=9.1 \mathrm{~Hz}, 1 \mathrm{H}), 4.97(\mathrm{~d}$, $J=7.1 \mathrm{~Hz}, 1 \mathrm{H}), 4.31(\mathrm{dd}, J=12.3,5.2 \mathrm{~Hz}, 1 \mathrm{H}), 4.18(\mathrm{dd}, J=$ 12.3, $2.5 \mathrm{~Hz}, 1 \mathrm{H}), 3.85-3.79(\mathrm{~m}, 1 \mathrm{H}), 3.79(\mathrm{~s}, 3 \mathrm{H}), 2.10$ (s, 3 $\mathrm{H}), 2.09(\mathrm{~s}, 3 \mathrm{H}), 2.06(\mathrm{~s}, 3 \mathrm{H}), 2.05(\mathrm{~s}, 3 \mathrm{H}) .{ }^{13} \mathrm{C}$ NMR $(75$ $\left.\mathrm{MHz}, \mathrm{CDCl}_{3}\right) \delta 170.6,170.2,169.4,169.3,155.8,150.9,118.7$ $(2 \mathrm{C}), 114.6(2 \mathrm{C}), 100.3,72.8,72.0,71.3,68.3,62.0,55.6$, $20.7(2 \mathrm{C}), 20.6(2 \mathrm{C})$.
1-(4'-Thiomethyl)-2,3,4,6-tetra-O-acetyl- $\alpha$-Dglucopyranoside $3 \mathrm{c}$.

The title compound was prepared according to the general procedure from commercially available 2,3,4,6-tetraO-acetyl-D-glucopyranose (174 mg, $\alpha: \beta=1: 3$ ) and $4^{-}$ thiomethylphenylboronic acid (250 $\mathrm{mg}$ ) and was purified on silica gel chromatography (cyclohexane/EtOAc, from 90/10 to 70/30) to afford $34 \mathrm{mg}$ of the $\alpha$ anomer and 82 $\mathrm{mg}$ of the $\beta$ anomer as amorphous white solids. Global yield: $49 \%$ (o.24 mmol, $115 \mathrm{mg}$ ).

1-(4'-Thiomethyl)-2,3,4,6-tetra-O-acetyl- $\alpha$-Dglucopyranoside.

${ }^{1} \mathrm{H}$ NMR (300 MHz, $\left.\mathrm{CDCl}_{3}\right) \delta$ 7.27-7.24 (m, $\left.2 \mathrm{H}\right)$, 7.05-7.03 (m, $2 \mathrm{H}), 5.73-5.66(\mathrm{~m}, 2 \mathrm{H}), 5.16(\mathrm{t}, J=9.7 \mathrm{~Hz}, 1 \mathrm{H}), 5.04$ $(\mathrm{dd}, J=10.2,3.7 \mathrm{~Hz}, 1 \mathrm{H}), 4.26(\mathrm{dd}, J=11.9,4.2 \mathrm{~Hz}, 1 \mathrm{H})$, 4.12 (ddd, $J=10.3,4.4,2.2 \mathrm{~Hz}, 1 \mathrm{H}$ ), 4.07 (dd, $J=12.3,2.3$ $\mathrm{Hz}, 1 \mathrm{H}), 2.47(\mathrm{~s}, 3 \mathrm{H}), 2.07-2.05(\mathrm{~m}, 12 \mathrm{H}) .{ }^{13} \mathrm{C}$ NMR $(75$ $\left.\mathrm{MHz}, \mathrm{CDCl}_{3}\right) \delta$ 170.5, 170.1 (2 C), 169.5, 154.3, 132.1, 129.3 (2 C), 117.3 ( 2 C), 94.4, 70.4, 70.0, 68.3, 68.0, 61.6, 20.7, 20.6 $(3 \mathrm{C}), 17 \cdot 3$. HRMS (ESI) calculated for $\mathrm{C}_{21} \mathrm{H}_{26} \mathrm{O}_{10} \mathrm{NaS}[\mathrm{M}+$ $\mathrm{Na}]^{+}$493.1144, found 493.1136. $[\alpha]_{\mathrm{D}}{ }^{13}+160.7^{\circ}$ (c 0.28 , $\mathrm{MeOH})$. FT-IR $\mathrm{v}_{\max } / \mathrm{cm}^{-1}: 1733,1493,1368,1222,1034,816$, 604, 559 .

1-(4'-Thiomethyl)-2,3,4,6-tetra-O-acetyl- $\beta$ - $D$ glucopyranoside.

${ }^{1} \mathrm{H}$ NMR (300 $\left.\mathrm{MHz}, \mathrm{CDCl}_{3}\right) \delta$ 7.26-7.23 (m, $2 \mathrm{H}$ ), 6.97-6.94 $(\mathrm{m}, 2 \mathrm{H}), 5.36-5.22(\mathrm{~m}, 1 \mathrm{H}), 5.17($ app t, $J=9.2 \mathrm{~Hz}, 1 \mathrm{H})$, $5.05(\mathrm{~d}, J=7.3 \mathrm{~Hz}, 1 \mathrm{H}), 4.30(\mathrm{dd}, J=12.3,5.3 \mathrm{~Hz}, 1 \mathrm{H}), 4.18$ (dd, $J=12.3,2.5 \mathrm{~Hz}, 1 \mathrm{H}), 3.86$ (ddd, $J=9.8,5.3,2.5 \mathrm{~Hz}, 1$ H), 2.46 (s, $3 \mathrm{H}), 2.09(\mathrm{~s}, 3 \mathrm{H}), 2.07(\mathrm{~s}, 3 \mathrm{H}), 2.06(\mathrm{~s}, 3 \mathrm{H})$, $2.05(\mathrm{~s}, 3 \mathrm{H}) .{ }^{13} \mathrm{C} \mathrm{NMR}\left(75 \mathrm{MHz}, \mathrm{CDCl}_{3}\right) \delta 170.5,170.2$, 169.3, 169.2, 155.0, 132.5, 129.2 (2 C), 117.8 (2 C), 99.3, 72.7, 72.1, 71.2, 68.3, 61.9, 20.7, 20.6 (3 C), 17.2. HRMS (ESI) calculated for $\mathrm{C}_{21} \mathrm{H}_{26} \mathrm{O}_{10} \mathrm{NaS}[\mathrm{M}+\mathrm{Na}]^{+}$493.1144, found 493.1138. $[\alpha]_{D}{ }^{13}-21.6^{\circ}(c \quad 0.37, \mathrm{MeOH})$. FT-IR $\mathrm{v}_{\max } / \mathrm{cm}^{-1}$ : 1740, 1493, 1366, 1218, 1059, 1033, 818, 699, 642.

\section{1-(4'-vinyl)-phenyl-2,3,4,6-tetra-O-acetyl-D-}

glucopyranoside $3 \mathrm{~d}$.

The title compound was prepared according to the general procedure from commercially available 2,3,4,6-tetraO-acetyl-D-glucopyranose (174 mg, $\alpha: \beta=1: 3$ ) and 4vinylphenylboronic acid $(222 \mathrm{mg}$ ) and was purified on silica gel chromatography (cyclohexane/EtOAc, from 9o/10 to 70/50) to afford $62 \mathrm{mg}$ of the $\alpha$ anomer and 126 $\mathrm{mg}$ of the $\beta$ anomer as amorphous white solids. Global yield: $84 \%$ (o.42 mmol, $189 \mathrm{mg}$ ).

\section{1-(4'-vinyl)-phenyl-2,3,4,6-tetra-O-acetyl- $\alpha$ - $D$ - glucopyranoside. $^{17}$}

${ }^{1} \mathrm{H}$ NMR (300 MHz, $\mathrm{CDCl}_{3}$ ) $\delta$ 7.35-7.32 (m, $2 \mathrm{H}$ ), 7.04-7.02 $(\mathrm{m}, 2 \mathrm{H}), 6.65(\mathrm{dd}, J=17.6,10.9 \mathrm{~Hz}, 1 \mathrm{H}), 5.73-5.60(\mathrm{~m}, 3$ H), $5.18-5.11(\mathrm{~m}, 2 \mathrm{H}), 5.03(\mathrm{dd}, J=10.2,3.7 \mathrm{~Hz}, 1 \mathrm{H}), 4.13-$ 4.01 (m, $2 \mathrm{H}), 4.23(\mathrm{dd}, J=12.1,4.4 \mathrm{~Hz}, 1 \mathrm{H}), 2.05(\mathrm{~s}, 3 \mathrm{H})$, $2.04(\mathrm{~s}, 3 \mathrm{H}), 2.03(\mathrm{~s}, 3 \mathrm{H}), 2.02(\mathrm{~s}, 3 \mathrm{H}) .{ }^{13} \mathrm{C}$ NMR $(75 \mathrm{MHz}$, $\left.\mathrm{CDCl}_{3}\right) \delta 170.5,170.1(2 \mathrm{C}), 169.5,155.7,135.8,132.7,127.4(2$ 
C), 116.6 (2 C), 112.9, 94.2, 70.4, 70.0, 68.3, 68.1, 61.6, 20.7, $20.6(3 \mathrm{C})$.

1-(4'-vinyl)-phenyl-2,3,4,6-tetra-O-acetyl- $\beta$-Dglucopyranoside. ${ }^{17}$

${ }^{1} \mathrm{H}$ NMR (300 MHz, $\mathrm{CDCl}_{3}$ ) $\delta$ 7.34-7.31 (m, $2 \mathrm{H}$ ), 6.95-6.92 $(\mathrm{m}, 2 \mathrm{H}), 6.65(\mathrm{dd}, J=17.6,10.9 \mathrm{~Hz}, 1 \mathrm{H}), 5.63(\mathrm{~d}, J=17.6$ $\mathrm{Hz}, 1 \mathrm{H}), 5.32-5.22(\mathrm{~m}, 2 \mathrm{H})$, 5.22-5.12 (m, $2 \mathrm{H})$, 5.08-5.06 (m, $1 \mathrm{H}), 4.28(\mathrm{dd}, J=12.3,5.3 \mathrm{~Hz}, 1 \mathrm{H}), 4.16(\mathrm{dd}, J=12.3$, $2.5 \mathrm{~Hz}, 1 \mathrm{H})$, 3.91-3.78 (m, $1 \mathrm{H}), 2.06$ (s, $3 \mathrm{H}), 2.04(\mathrm{~s}, 3 \mathrm{H})$, $2.03(\mathrm{~s}, 3 \mathrm{H}), 2.02(\mathrm{~s}, 3 \mathrm{H}) \cdot{ }^{13} \mathrm{C} \mathrm{NMR}\left(75 \mathrm{MHz} \mathrm{CDCl}_{3}\right) \delta$ 170.5, 170.2, 169.3, 169.2, 156.5, 135.8, 133.1, 127.4 (2 C), 117.0 (2 C), 113.0, 99.1, 72.7, 72.1, 71.2, 68.3, 61.9, 20.7, 20.6 (3 C).

\section{1-(3'-Methoxy)-phenyl-2,3,4,6-tetra-O-acetyl-D- glucopyranoside 3 f.}

The title compound was prepared according to the general procedure from commercially available 2,3,4,6-tetraO-acetyl-D-glucopyranose (174 mg, $\alpha: \beta=1: 3$ ) and $3^{-}$ methoxyphenylboronic acid $(227 \mathrm{mg})$ and was purified on silica gel chromatography (cyclohexane/EtOAc, from 90/10 to 70/30) to afford $42 \mathrm{mg}$ of the $\alpha$ anomer and 134 $\mathrm{mg}$ of the $\beta$ anomer as amorphous white solids. Global yield: $77 \%$ (o.38 mmol, $175 \mathrm{mg}$ ).

\section{1-(3'-Methoxy)-phenyl-2,3,4,6-tetra-O-acetyl- $\alpha$-D- glucopyranoside.}

${ }^{1} \mathrm{H}$ NMR (300 MHz, $\mathrm{CDCl}_{3}$ ) $\delta 7.19$ (app t, $J=8.1 \mathrm{~Hz}, 1 \mathrm{H}$ ), 6.69-6.6o (m, $3 \mathrm{H}$ ), 5.79-5.6o (m, $2 \mathrm{H}$ ), 5.14 (app t, $J=9.7$ $\mathrm{Hz}, 1 \mathrm{H}), 5.03(\mathrm{dd}, J=10.3,3.6 \mathrm{~Hz}, 1 \mathrm{H}), 4.25(\mathrm{dd}, J=12.1$, $4.5 \mathrm{~Hz}, 1 \mathrm{H}), 4.16-4.08(\mathrm{~m}, 1 \mathrm{H}), 4.05(\mathrm{dd}, J=12.1,2.2 \mathrm{~Hz}, 1$ $\mathrm{H}), 3.79(\mathrm{~s}, 3 \mathrm{H}), 2.06(\mathrm{~m}, 6 \mathrm{H}), 2.05(\mathrm{~s}, 3 \mathrm{H}), 2.04(\mathrm{~s}, 5 \mathrm{H})$. ${ }^{13} \mathrm{C}$ NMR (75 MHz, $\mathrm{CDCl}_{3}$ ) $\delta$ 170.5, 170.1 (2 C), 169.6, 16o.8, 157.2, 130.1, 108.7, 108.5, 103.0, 94.2, 70.4, 70.1, 68.4, 68.0, $61.6,55.4,20.7,20.6$ (3 C). HRMS (ESI) calculated for $\mathrm{C}_{21} \mathrm{H}_{26} \mathrm{O}_{11} \mathrm{Na}[\mathrm{M}+\mathrm{Na}]^{+}$477.1373, found 477.1378. $[\alpha]_{\mathrm{D}}{ }^{14}$ $+146.3^{\circ}$ (c o.21, MeOH). FT-IR $v_{\max } / \mathrm{cm}^{-1}: 1739,1593,1493$, $1368,1223,1033,787,691,603$.

\section{1-(3'-Methoxy)-phenyl-2,3,4,6-tetra-O-acetyl- $\beta$-D- glucopyranoside. ${ }^{15}$}

${ }^{1} \mathrm{H}$ NMR (30o MHz, $\mathrm{CDCl}_{3}$ ) $\delta 7.18$ (app t, $J=8.2 \mathrm{~Hz}, 1 \mathrm{H}$ ), 6.67-6.52 (m, $2 \mathrm{H})$, 5.35-5.21 (m, $2 \mathrm{H}), 5.19-5.11(\mathrm{~m}, 1 \mathrm{H})$, $5.07(\operatorname{app} d, J=7.3 \mathrm{~Hz}, 1 \mathrm{H}), 4.27(\mathrm{dd}, J=12.3,5.4 \mathrm{~Hz}, 1 \mathrm{H})$, 4.16 (dd, $J=12.3,2.5 \mathrm{~Hz}, 1 \mathrm{H}$ ), 3.85 (ddd, $J=9.8,5.4,2.5$ $\mathrm{Hz}, 1 \mathrm{H}), 3.78(\mathrm{~s}, 3 \mathrm{H}), 2.07(\mathrm{~s}, 3 \mathrm{H}), 2.05(\mathrm{~s}, 3 \mathrm{H}), 2.04(\mathrm{~s}, 3$ $\mathrm{H}), 2.03(\mathrm{~s}, 3 \mathrm{H}) .{ }^{13} \mathrm{C}$ NMR $\left(75 \mathrm{MHz}, \mathrm{CDCl}_{3}\right) \delta$ 170.6, 170.2, 169.4, 169.3, 160.8, 158.0, 130.0, 110.0, 108.9, 108.6, 103.6, 99.0, 72.8, 72.1, 71.2, 68.4, 62.0, 55.4, $20.6\left({ }_{4} \mathrm{C}\right)$.

\section{1-(3',5'-dimethyl)-phenyl-2,3,4,6-tetra-O-acetyl-D- glucopyranoside $3 \mathrm{~g}$.}

The title compound was prepared according to the general procedure from commercially available 2,3,4,6-tetraO-acetyl-D-glucopyranose (174 mg, $\alpha: \beta=1: 3$ ) and 3,5dimethylphenylboronic acid $(225 \mathrm{mg}$ ) and was purified on silica gel chromatography (cyclohexane/EtOAc, from
90/10 to 70/30) to afford $52 \mathrm{mg}$ of the $\alpha$ anomer and 106 $\mathrm{mg}$ of the $\beta$ anomer as amorphous white solids. Global yield: $70 \%$ (o.35 mmol, $158 \mathrm{mg}$ ).

1-(3', 5'-dimethyl)-phenyl-2,3,4,6-tetra-O-acetyl- $\alpha$ - Dglucopyranoside.

${ }^{1} \mathrm{H}$ NMR (300 $\left.\mathrm{MHz}, \mathrm{CDCl}_{3}\right) \delta 6.7 \mathrm{o}(\mathrm{m}, 3 \mathrm{H}), 5.69$ (app t, $J=$ $9.7 \mathrm{~Hz}, 1 \mathrm{H}), 5.7 \mathrm{O}(\mathrm{d}, J=3.7 \mathrm{~Hz}, 1 \mathrm{H}), 5.15($ app t, $J=9.8 \mathrm{~Hz}$, $1 \mathrm{H}), 5.02(\mathrm{dd}, J=10.2,3.7 \mathrm{~Hz}, 1 \mathrm{H}), 4.26(\mathrm{dd}, J=12.2,4.5$ $\mathrm{Hz}, 1 \mathrm{H}), 4.13$ (ddd, $J=10.1,4.5,2.1 \mathrm{~Hz}, 1 \mathrm{H}), 4.05$ (dd, $J=$ 12.2, $2.3 \mathrm{~Hz}, 1 \mathrm{H}), 2.29(\mathrm{~s}, 6 \mathrm{H}), 2.05-2.03(\mathrm{~m}, 12 \mathrm{H}) .{ }^{13} \mathrm{C}$ NMR $\left(75 \mathrm{MHz}, \mathrm{CDCl}_{3}\right) \delta 170.5,170.1(2 \mathrm{C}), 169.6,156.1$, 139.5, 124.7, 114.2 (2 C), 94.1, 70.5, 70.1, 68.4, 67.9, 61.7, 21.4 (2 C), 20.7, 20.6 (3 C). HRMS (ESI) calculated for $\mathrm{C}_{22} \mathrm{H}_{28} \mathrm{O}_{10} \mathrm{Na}[\mathrm{M}+\mathrm{Na}]^{+} 475.1580$, found 475.1574. $[\alpha]_{\mathrm{D}}{ }^{14}$ $+160.9^{\circ}$ (c o.23, MeOH). FT-IR $v_{\max } / \mathrm{cm}^{-1}: 1743,1593,1367$, 1214, 1172, 1155, 1033, 839, 600 .

1-(3,5'-dimethyl)-phenyl-2,3,4,6-tetra-O-acetyl- $\beta-D$ glucopyranoside.

${ }^{1} \mathrm{H}$ NMR (300 MHz, $\left.\mathrm{CDCl}_{3}\right) \delta 6.71(\mathrm{~m}, 1 \mathrm{H}), 6.61(\mathrm{~m}, 2 \mathrm{H})$, 5.32-5.21 (m, $2 \mathrm{H}), 5.14($ app t, $J=9.4 \mathrm{~Hz}, 1 \mathrm{H}), 5.05$ (app d, $J=7.1 \mathrm{~Hz}, 1 \mathrm{H}), 4.26(\mathrm{dd}, J=12.2,5.5 \mathrm{~Hz}, 1 \mathrm{H}), 4.18(\mathrm{dd}, J=$ $12.2,2.6 \mathrm{~Hz}, 1 \mathrm{H}$ ), 3.87 (ddd, $J=9.9,5.5,2.5 \mathrm{~Hz}, 1 \mathrm{H}$ ), 2.28 (s, $6 \mathrm{H}), 2.08(\mathrm{~s}, 3 \mathrm{H}), 2.05(\mathrm{~s}, 3 \mathrm{H}), 2.04(\mathrm{~s}, 3 \mathrm{H}), 2.03(\mathrm{~s}, 3$ $\mathrm{H}) .{ }^{13} \mathrm{C}$ NMR $\left(75 \mathrm{MHz}, \mathrm{CDCl}_{3}\right) \delta 170.5,170.2,169.4,169.3$, 156.9, 139.4, 125.0, 114.6 (2 C), 99.0, 72.8, 72.0, 71.2, 68.4, 62.2, 21.4 (2 C), 20.7, 20.6 (3 C). HRMS (ESI) calculated for $\mathrm{C}_{22} \mathrm{H}_{28} \mathrm{O}_{10} \mathrm{Na}[\mathrm{M}+\mathrm{Na}]^{+} 475.1580$, found $475.1579 .[\alpha]_{\mathrm{D}}{ }^{14}-$ $25.3^{\circ}$ (c 0.48, MeOH). FT-IR $v_{\max } / \mathrm{cm}^{-1}: 1751,1595,1367$, 1214, 1136, 1036, 954, 906, 846, 599.

\section{1-(4'-Bromo)-phenyl-2,3,4,6-tetra-O-acetyl-D-} glucopyranoside $3 \mathbf{h}$.

The title compound was prepared according to the general procedure from commercially available 2,3,4,6-tetraO-acetyl-D-glucopyranose (174 mg, $\alpha: \beta=1: 3$ ) and 4bromophenylboronic acid $(301 \mathrm{mg}$ ) and was purified on silica gel chromatography (cyclohexane/EtOAc, from 90/10 to 70/30) to afford $40 \mathrm{mg}$ of the $\alpha$ anomer and 139 $\mathrm{mg}$ of the $\beta$ anomer as amorphous white solids. Global yield: $71 \%$ (0.35 mmol, $178 \mathrm{mg})$.

\section{1-(4'-Bromophenyl)-2,3,4,6-tetra-O-acetyl- $\alpha$-D- glucopyranoside. ${ }^{16}$}

${ }^{1} \mathrm{H}$ NMR (300 $\mathrm{MHz}, \mathrm{CDCl}_{3}$ ) $\delta$ 7.42-7.39 (m, $2 \mathrm{H}$ ), 6.99-6.96 $(\mathrm{m}, 2 \mathrm{H}), 5.73-5.59(\mathrm{~m}, 1 \mathrm{H}), 5.14($ app t, $J=9.9 \mathrm{~Hz}, 1 \mathrm{H})$, $5.02(\mathrm{dd}, J=10.3,3.7 \mathrm{~Hz}, 1 \mathrm{H}), 4.23$ (dd, $J=12.3,4.7 \mathrm{~Hz}, 1$ H), 4.12-3.99 (m, $1 \mathrm{H}), 2.06(\mathrm{~s}, 3 \mathrm{H}), 2.04(\mathrm{~s}, 3 \mathrm{H}), 2.03$ (s, 6 H). ${ }^{13} \mathrm{C}$ NMR (75 MHz, $\left.\mathrm{CDCl}_{3}\right) \delta 170.5,170.1$ (2 C), 169.5 , 155.1, 132.6 (2 C), 118.4 (2 C), 115.6, 94.4, 70.4, 69.9, 68.2, 61.6, 20.7, 20.6 (3C).

\section{1-(4'-Bromophenyl)-2,3,4,6-tetra-O-acetyl- $\beta$ - $D$ -} glucopyranoside. ${ }^{18}$

${ }^{1} \mathrm{H}$ NMR (300 MHz, $\left.\mathrm{CDCl}_{3}\right) \delta$ 7.40-7.37 (m, $\left.2 \mathrm{H}\right), 6.89-6.86$ (m, $2 \mathrm{H}), 5.34-5.19(\mathrm{~m}, 2 \mathrm{H}), 5.15($ app t, $J=9.2 \mathrm{~Hz}, 1 \mathrm{H})$, 
$5.03(\mathrm{~d}, J=7.3 \mathrm{~Hz}, 1 \mathrm{H}), 4.27$ (dd, $J=12.3,5.3 \mathrm{~Hz}, 1 \mathrm{H}), 4.15$ (dd, $J=12.3,2.5 \mathrm{~Hz}, 1 \mathrm{H}$ ), 3.84 (ddd, $J=10.0,5.4,2.5 \mathrm{~Hz}, 1$ H), 2.07 (s, $3 \mathrm{H}), 2.05$ (s, $3 \mathrm{H}), 2.04(\mathrm{~s}, 3 \mathrm{H}), 2.02$ (s, $3 \mathrm{H})$. ${ }^{13} \mathrm{C} \mathrm{NMR}\left(75 \mathrm{MHz}, \mathrm{CDCl}_{3}\right) \delta 170.5,170.2,169.3,169.2,155.9$, 132.5 (2 C), 118.9 (2 C), 115.9, 99.1, 72.6, 72.1, 71.1, 68.2, 61.9, $20.7(2 \mathrm{C}), 20.6(2 \mathrm{C})$.

\section{1-(4'-Fluorophenyl)-2,3,4,6-tetra-O-acetyl-D- glucopyranoside $3 \mathbf{i}$.}

The title compound was prepared according to the general procedure from commercially available 2,3,4,6-tetraO-acetyl-D-glucopyranose (174 $\mathrm{mg}, \alpha: \beta=1: 3$ ) and $4^{-}$ fluorophenylboronic acid (210 $\mathrm{mg}$ ) and was purified on silica gel chromatography (cyclohexane/EtOAc, from 90/10 to 70/30) to afford $22 \mathrm{mg}$ of the $\alpha$ anomer and 105 mg of the $\beta$ anomer as amorphous white solids. Global yield: $58 \%$ (o.29 mmol, $128 \mathrm{mg}$ ).

\section{1-(4'-Fluorophenyl)-2,3,4,6-tetra-O-acetyl- $\alpha$-D- glucopyranoside. ${ }^{16}$}

${ }^{1} \mathrm{H}$ NMR (300 $\mathrm{MHz}, \mathrm{CDCl}_{3}$ ) $\delta$ 7.06-6.95 (m, $4 \mathrm{H}$ ), 5.74-5.57 (m, $2 \mathrm{H}), 5.13$ (app t, $J=9.6 \mathrm{~Hz}, 1 \mathrm{H}), 5.01(\mathrm{dd}, J=10.3,3.7$ $\mathrm{Hz}, 1 \mathrm{H}), 4.23$ (dd, $J=11.8,4.4 \mathrm{~Hz}, 1 \mathrm{H}), 4.16-3.99(\mathrm{~m}, 2 \mathrm{H})$, 2.06-2.03 (m, 12 H). $\left.{ }^{13} \mathrm{C} \mathrm{NMR} \mathrm{(75} \mathrm{MHz,} \mathrm{CDCl}_{3}\right) \delta$ 170.5, $170.1(2 \mathrm{C}), 169.6,158.6\left(J_{13 C\{F\}}=241.4 \mathrm{~Hz}\right), 152.2$, 118.0 $\left(J_{13 C\{F\}}\right.$ $=7.9 \mathrm{~Hz}, 2 \mathrm{C})$, $116.1\left(J_{13 C\{F\}}=23.1 \mathrm{~Hz}, 2 \mathrm{C}\right), 94.9,70.5$, 70.0, 68.3, 68.1, 61.6, 20.7, 20.6 (3 C). ${ }^{19} \mathrm{~F}\left(188 \mathrm{MHz} \mathrm{CDCl}_{3}\right) \delta-$ 120.6 .

\section{1-(4'-Fluorophenyl)-2,3,4,6-tetra-O-acetyl- $\beta$ - $D$ -} glucopyranoside. ${ }^{15}$

${ }^{1} \mathrm{H}$ NMR (300 MHz, $\mathrm{CDCl}_{3}$ ) $\delta$ 7.00-6.95 (m, $4 \mathrm{H}$ ), 5.31-5.20 (m, $2 \mathrm{H}), 5.15$ (app t, $J=9.2 \mathrm{~Hz}, 1 \mathrm{H}), 4.98$ (d, $J=7.0 \mathrm{~Hz}, 1$ H), 4.28 (dd, $J=12.3,5.3 \mathrm{~Hz}, 1 \mathrm{H}), 4.16(\mathrm{dd}, J=12.3,2.5 \mathrm{~Hz}$, $1 \mathrm{H}), 3.81$ (ddd, $J=10.0,5.3,2.5 \mathrm{~Hz}, 1 \mathrm{H}), 2.07$ (s, $3 \mathrm{H}), 2.06$ $(\mathrm{s}, 3 \mathrm{H}), 2.03(\mathrm{~s}, 3 \mathrm{H}), 2.02(\mathrm{~s}, 3 \mathrm{H}) .{ }^{13} \mathrm{C} \mathrm{NMR}(75 \mathrm{MHz}$, $\left.\mathrm{CDCl}_{3}\right) \delta_{170.5}, 170.2,169.3,169.2,158.8\left(J_{13 C\{F\}}=241.5 \mathrm{~Hz}\right)$, 152.9, $118.8\left(J_{13 C\{F\}}=8.5 \mathrm{~Hz}, 2 \mathrm{C}\right)$, $116.0\left(J_{13 C\{F\}}=23.2 \mathrm{~Hz}, 2\right.$ C), 99.9, 72.7, 72.1, 71.2, 68.3, 61.9, 20.7, 20.6 (3 C). ${ }^{19} \mathrm{~F}(188$ $\left.\mathrm{MHz}, \mathrm{CDCl}_{3}\right) \delta-120.1$.

\section{1-(4'-Trifluoromethyl)-phenyl-2,3,4,6-tetra-O-acetyl- D-glucopyranoside $3 \mathbf{j}$.}

The title compound was prepared according to the general procedure from commercially available 2,3,4,6-tetraO-acetyl-D-glucopyranose (174 $\mathrm{mg}, \alpha: \beta=1: 3$ ) and $4^{-}$ trifluoromethylphenylboronic acid (286 mg) and was purified on silica gel chromatography (cyclohexane/EtOAc, from 90/10 to 70/30) to afford $36 \mathrm{mg}$ of the $\alpha$ anomer and $145 \mathrm{mg}$ of the $\beta$ anomer as amorphous white solids. Global yield: 74 \% (o.29 mmol, $182 \mathrm{mg}$ ).

1-(4'-Trifluoromethyl)-phenyl-2,3,4,6-tetra-O-acetyl- $\alpha$-Dglucopyranoside.

${ }^{1} \mathrm{H}$ NMR (300 MHz, $\mathrm{CDCl}_{3}$ ) $\delta$ 7.59-7.56 (m, $2 \mathrm{H}$ ), 7.19-7.17 $(\mathrm{m}, 2 \mathrm{H}), 5.80$ (d, $J=3.7 \mathrm{~Hz}, 1 \mathrm{H}), 5.69$ (app t, $J=9.8 \mathrm{~Hz}, 1$ H), 5.15 (app t, $J=9.7 \mathrm{~Hz}, 1 \mathrm{H}), 5.05(\mathrm{dd}, J=10.3,3.7 \mathrm{~Hz}, 1$ H), 4.23 (dd, $J=12.8,5.1 \mathrm{~Hz}, 1 \mathrm{H}), 4.06-4.03$ (m, $2 \mathrm{H}), 2.06$ (s, $3 \mathrm{H}), 2.05(\mathrm{~s}, 3 \mathrm{H}), 2.04(\mathrm{~s}, 3 \mathrm{H}), 2,02(\mathrm{~s}, 3 \mathrm{H}) .{ }^{13} \mathrm{C}$ NMR $\left(75 \mathrm{MHz} \mathrm{CDCl}_{3}\right) \delta 170.4,170.1$ (2 C), 169.5, 158.3, 127.1, 127.0, $125.3\left(\mathrm{q}, J_{13 C\{F\}}=33 \mathrm{~Hz}\right), 124.0\left(\mathrm{q}, J_{13 C\{F\}}=270 \mathrm{~Hz}\right)$, 116.5 (2 C), 94.0, 70.3, 69.9, 68.4, 68.2, 61.5, 20.6, 20.5 (3 C). ${ }^{19} \mathrm{~F}\left(188 \mathrm{MHz}, \mathrm{CDCl}_{3}\right) \delta$-61.9. HRMS (ESI) calculated for $\mathrm{C}_{21} \mathrm{H}_{23} \mathrm{O}_{10} \mathrm{NaF}_{3}[\mathrm{M}+\mathrm{Na}]^{+}$515.1141, found $515.1143 \cdot[\alpha]_{\mathrm{D}}{ }^{13}$ $+137.0^{\circ}$ (c 0.27, MeOH). FT-IR $\mathrm{v}_{\max } / \mathrm{cm}^{-1}: 1742,1330,1224$, $1123,1032,844,687,608$.

\section{1-(4'-Trifluoromethyl)-phenyl-2,3,4,6-tetra-O-acetyl- $\beta$ - $D$ - glucopyranoside. ${ }^{16}$}

${ }^{1} \mathrm{H}$ NMR (300 $\left.\mathrm{MHz}, \mathrm{CDCl}_{3}\right) \delta$ 7.57-7.55 (m, $2 \mathrm{H}$ ), 7.07-7.05 (m, $2 \mathrm{H}$ ), 5.34-5.25 (m, $2 \mathrm{H}), 5.20-5.14$ (m, $2 \mathrm{H}), 4.28$ (dd, $J$ $=12.3,5.4 \mathrm{~Hz}, 1 \mathrm{H}), 4.17(\mathrm{dd}, J=12.3,2.5 \mathrm{~Hz}, 1 \mathrm{H}), 3.89$ (ddd, $J=9.9,5.4,2.5 \mathrm{~Hz}, 1 \mathrm{H}), 2.06(\mathrm{~s}, 3 \mathrm{H}), 2.05(\mathrm{~s}, 6 \mathrm{H})$, $2.03(\mathrm{~s}, 3 \mathrm{H}) \cdot{ }^{13} \mathrm{C} \mathrm{NMR}\left(75 \mathrm{MHz}, \mathrm{CDCl}_{3}\right) \delta 170.4,170.1$, 169.3, 169.2, 159.0, 127.0 (2 C), 125.4 (q, $\left.J_{13 C\{F\}}=33 \mathrm{~Hz}\right)$, 124.0 $\left(\mathrm{q}, J_{13 C\{F\}}=270 \mathrm{~Hz}\right), 116.8(2 \mathrm{C}), 98.4,72.5,72.2,71.0,68.2$, 61.9, $20.6(2 \mathrm{C}), 20.5(2 \mathrm{C}) .{ }^{19} \mathrm{~F}\left(188 \mathrm{MHz}, \mathrm{CDCl}_{3}\right) \delta-61.9$.

1-(4'-trifluoromethoxy)-phenyl-2,3,4,6-tetra-O-acetylD-glucopyranoside $3 \mathbf{k}$.

The title compound was prepared according to the general procedure from commercially available 2,3,4,6-tetraO-acetyl-D-glucopyranose (174 $\mathrm{mg}, \alpha: \beta=1: 3$ ) and 4(trifluoromethoxy)phenylboronic acid (310 $\mathrm{mg}$ ) and was purified on silica gel chromatography (cyclohexane/EtOAc, from $90 / 10$ to $60 / 40$ ) to afford $39 \mathrm{mg}$ of the $\alpha$ anomer and $139 \mathrm{mg}$ of the $\beta$-anomer as amorphous white solids. Global yield: $70 \%$ (o.35 mmol, $178 \mathrm{mg})$.

1-(4'-trifluoromethoxy)-phenyl-2,3,4,6-tetra-O-acetyl- $\alpha$ - $D$ glucopyranoside.

${ }^{1} \mathrm{H}$ NMR (300 $\mathrm{MHz}, \mathrm{CDCl}_{3}$ ) $\delta$ 7.24-6.98 (m, $4 \mathrm{H}$ ), 5.71-5.64 $(\mathrm{m}, 2 \mathrm{H}), 5.14($ app t, $J=9.9 \mathrm{~Hz}, 1 \mathrm{H}), 5.03(\mathrm{dd}, J=10.3,3.7$ $\mathrm{Hz}, 1 \mathrm{H}), 4.23(\mathrm{dd}, J=12.3,4.8 \mathrm{~Hz}, 1 \mathrm{H}), 4.11-4.05(\mathrm{~m}, 2 \mathrm{H})$, $2.06(\mathrm{~s}, 3 \mathrm{H}), 2.05(\mathrm{~s}, 3 \mathrm{H}), 2.03(\mathrm{~s}, 3 \mathrm{H}), 2.02(\mathrm{~s}, 2 \mathrm{H}) .{ }^{13} \mathrm{C}$ NMR (75 MHz, $\left.\mathrm{CDCl}_{3}\right) \delta 170.4,170.1$ (2 C), 169.5, 154.4, $144.5,122.5,122.2(2 \mathrm{C}), 120.5\left(\mathrm{q}, J_{13 C\{F\}}=252 \mathrm{~Hz}\right), 117.6(2 \mathrm{C})$, 94.5, 70.4, 69.9, 68.3, 68.2, 61.6, 20.5 (4 C). ${ }^{19} \mathrm{~F}(188 \mathrm{MHz}$, $\left.\mathrm{CDCl}_{3}\right) \delta$-58.4. HRMS (ESI) calculated for $\mathrm{C}_{21} \mathrm{H}_{23} \mathrm{O}_{11} \mathrm{~F}_{3} \mathrm{Na}$ $[\mathrm{M}+\mathrm{Na}]^{+}$531.1090, found 531.1082. $[\alpha]_{\mathrm{D}}{ }^{14}+128.6^{\circ}$ (c o.21, $\mathrm{MeOH})$. FT-IR $\mathrm{v}_{\max } / \mathrm{cm}^{-1}: 1745,1505,1368,1213,1192,1161$, 1033, 918, 801, 601 .

1-(4'-trifluoromethoxy)-phenyl-2,3,4,6-tetra-O-acetyl- $\beta$ - $D$ glucopyranoside. ${ }^{16}$

${ }^{1} \mathrm{H}$ NMR (300 $\mathrm{MHz}, \mathrm{CDCl}_{3}$ ) $\delta$ 7.17-7.14 (m, $2 \mathrm{H}$ ), 7.01-6.98 (m, $2 \mathrm{H}), 5 \cdot 33-5.23(\mathrm{~m}, 2 \mathrm{H}), 5.16($ app t, $J=9.4 \mathrm{~Hz}, 1 \mathrm{H})$, $5.06(\mathrm{~d}, J=7.2 \mathrm{~Hz}, 1 \mathrm{H}), 4.28(\mathrm{dd}, J=12.3,5.3 \mathrm{~Hz}, 1 \mathrm{H}), 4.17$ (dd, $J=12.3,2.5 \mathrm{~Hz}, 1 \mathrm{H}$ ), 3.85 (ddd, $J=10.0,5.4,2.6 \mathrm{~Hz}, 1$ $\mathrm{H}), 2.06$ (m, 6 H), 2.05 (s, $3 \mathrm{H}), 2.03$ (s, $3 \mathrm{H}) .{ }^{13} \mathrm{C}$ NMR (75 $\left.\mathrm{MHz} \mathrm{CDCl}_{3}\right) \delta_{170.4}, 170.1,169.3,169.2,155.1,144.7,122.4(2$ C), $120.4\left(J_{C-F}=255 \mathrm{~Hz}\right), 118.1(2 \mathrm{C}), 99.2,72.6,72.2,71.1$, 68.3, 61.9, 20.5 (4 C). ${ }^{19} \mathrm{~F}\left(188 \mathrm{MHz}, \mathrm{CDCl}_{3}\right) \delta-58.3$.

1-(4'-acetyl)-phenyl-2,3,4,6-tetra-O-acetyl-Dglucopyranoside 31 . 
The title compound was prepared according to the general procedure from commercially available 2,3,4,6-tetraO-acetyl-D-glucopyranose (174 $\mathrm{mg}, \alpha: \beta=1: 3$ ) and 4acetylphenylboronic acid (245 $\mathrm{mg}$ ) and was purified on silica gel chromatography (cyclohexane/EtOAc, from 90/10 to 50/50) to afford $32 \mathrm{mg}$ of the $\alpha$ anomer and 95 mg of the $\beta$ anomer as amorphous white solids. Global yield: $74 \%$ (0.37 mmol, $172 \mathrm{mg}$ ).

\section{1-(4'-acetyl)-phenyl-2,3,4,6-tetra-O-acetyl- $\alpha$-D- glucopyranoside.}

${ }^{1} \mathrm{H}$ NMR (300 $\mathrm{MHz} \mathrm{CDCl}_{3}$ ) $\delta$ 7.95-7.93 (m, $2 \mathrm{H}$ ), 7.16-7.13 $(\mathrm{m}, 2 \mathrm{H}), 5.82(\mathrm{~d}, J=3.6 \mathrm{~Hz}, 1 \mathrm{H}), 5.70$ (app t, $J=9.8 \mathrm{~Hz}, 1$ H), 5.16 (app t, $J=9.9 \mathrm{~Hz}, 1 \mathrm{H}), 5.06(\mathrm{dd}, J=10.2,3.6 \mathrm{~Hz}, 1$ H), 4.24 (dd, $J=12.3,4.5 \mathrm{~Hz}, 1 \mathrm{H}$ ), 4.09-4.02 (m, $2 \mathrm{H}), 2.57$ (s, $3 \mathrm{H}), 2,06-2.03(\mathrm{~m}, 12 \mathrm{H}) .{ }^{13} \mathrm{C} \mathrm{NMR}\left(75 \mathrm{MHz}, \mathrm{CDCl}_{3}\right) \delta$ 196.6, 170.4, 170.1 (2 C), 169.5, 159.6, 132.3, 130.5 (2 C), 116.2 (2 C), 93.9, 70.3, 69.9, 68.4, 68.2, 61.5, 26.4, 20.7, 20.6 (2 C). HRMS (ESI) calculated for $\mathrm{C}_{22} \mathrm{H}_{26} \mathrm{O}_{11} \mathrm{Na}[\mathrm{M}+\mathrm{Na}]^{+}$ 489.1373, found 489.1365. $[\alpha]_{D}{ }^{13}+177.8^{\circ}$ (c o.23, MeOH). FT-IR $v_{\max } / \mathrm{cm}^{-1}: 1736,1677,1600,1360,1223,1033,829,761$, 590.

\section{1-(4'-acetyl)-phenyl-2,3,4,6-tetra-O-acetyl- $\beta$ - $D$ - glucopyranoside. ${ }^{19}$}

${ }^{1} \mathrm{H}$ NMR (300 $\mathrm{MHz}, \mathrm{CDCl}_{3}$ ) $\delta$ 7.96-7.93 (m, $2 \mathrm{H}$ ), 7.06-7.03 (m, $2 \mathrm{H}$ ), 5.37-5.28 (m, $2 \mathrm{H})$, 5.22-5.16 (m, $2 \mathrm{H}), 4.30$ (dd, $J$ = 12.3, $5.4 \mathrm{~Hz}, 1 \mathrm{H}), 4.19(\mathrm{dd}, J=12.3,2.5 \mathrm{~Hz}, 1 \mathrm{H}), 3.96-3.91$ $(\mathrm{m}, 1 \mathrm{H}), 2.58(\mathrm{~s}, 3 \mathrm{H}), 2.09-2.05(\mathrm{~m}, 12 \mathrm{H}) .{ }^{13} \mathrm{C}$ NMR $(75$ $\left.\mathrm{MHz} \mathrm{CDCl}_{3}\right) \delta 196.6,170.5,170.1,169.3,169.2,160.2,132.4$, 130.5 (2 C), 116.3 (2 C), 98.2, 72.6, 72.3, 71.1, 68.2, 61.9, 26.4, 20.7, $20.6(2 \mathrm{C})$.

\section{1-(3'-Iodo)-phenyl-2,3,4,6-tetra-O-acetyl-D- glucopyranoside $\mathbf{3 m}$.}

The title compound was prepared according to the general procedure from commercially available 2,3,4,6-tetraO-acetyl-D-glucopyranose (174 $\mathrm{mg}, \alpha: \beta=1: 3$ ) and $3^{-}$ iodophenylboronic acid (373 $\mathrm{mg}$ ) and was purified on silica gel chromatography (cyclohexane/EtOAc, from 90/10 to 70/30) to afford $54 \mathrm{mg}$ of the $\alpha$ anomer and 164 mg of the $\beta$ anomer as amorphous white solids. Global yield: $79 \%$ (0.4 mmol, $217 \mathrm{mg}$ ).

\section{1-(3'-Iodo)-phenyl-2,3,4,6-tetra-O-acetyl- $\alpha$ - D- glucopyranoside. $^{20}$}

${ }^{1} \mathrm{H}$ NMR (300 $\left.\mathrm{MHz}, \mathrm{CDCl}_{3}\right) \delta 7.47(\mathrm{~m}, 1 \mathrm{H}), 7.42-7.39(\mathrm{~m}, 1$ H), 7.07-6.99 (m, 2 H), 5.69 (d, $J=3.4$ Hz, 1 H), 5.66 (app t, $J=9.9 \mathrm{~Hz}, 1 \mathrm{H}), 5.13(\mathrm{app} t, J=9.9 \mathrm{~Hz}, 1 \mathrm{H}), 5.02(\mathrm{dd}, J=$ 10.2, 3.7 Hz, $1 \mathrm{H}), 4.24$ (dd, $J=12.5,5.1 \mathrm{~Hz}, 1 \mathrm{H}), 4.14-3.88$ (m, $2 \mathrm{H}), 2.06(\mathrm{~m}, 6 \mathrm{H}), 2.04(\mathrm{~s}, 3 \mathrm{H}), 2.03(\mathrm{~s}, 3 \mathrm{H}) .{ }^{13} \mathrm{C}$ NMR (75 MHz, $\left.\mathrm{CDCl}_{3}\right) \delta 170.5,170.1$ (2 C), 169.5, 156.4, 132.3, 131.0, 125.8, 116.0, 94.3, 94.2, 70.3, 69.9, 68.3, 68.2, 61.6, 20.7 (2 C), 20.6 (2 C).

1-(3'-Iodo)-phenyl-2,3,4,6-tetra-O-acetyl- $\beta$ - $D$ glucopyranoside. ${ }^{18}$
${ }^{1} \mathrm{H}$ NMR (30o $\mathrm{MHz}, \mathrm{CDCl}_{3}$ ) $\delta$ 7.45-7.32 (m, $2 \mathrm{H}$ ), 7.04-6.93 (m, $2 \mathrm{H}), 5.32-5.21(\mathrm{~m}, 2 \mathrm{H}), 5.12$ (app t, $J=9.3 \mathrm{~Hz}, 1 \mathrm{H})$, 5.05 (app d, $J=7.1 \mathrm{~Hz}, 1 \mathrm{H}$ ), 4.32-4.13 (m, 2 H), 3.88 (ddd, $J$ = 10.2, 5.8, 2.7 Hz, 1 H), 2.11 (s, $3 \mathrm{H}), 2.05$ (s, $3 \mathrm{H}), 2.04$ (s, 3 $\mathrm{H})$, $2.02(\mathrm{~s}, 3 \mathrm{H}) .{ }^{13} \mathrm{C} \mathrm{NMR}\left(75 \mathrm{MHz} \mathrm{CDCl}_{3}\right) \delta 170.6,170.1$, $169.4,169.2,157.1,132.5,130.9,125.9,116.6,98.8,94.0,72.6$, 72.2, 71.1, 68.3, 62.1, 20.8, 20.6 (3 C).

\section{1-(3'-Nitro)-phenyl-2,3,4,6-tetra-O-acetyl-D- glucopyranoside $3 n$.}

The title compound was prepared according to the general procedure from commercially available 2,3,4,6-tetraO-acetyl-D-glucopyranose (174 mg, $\alpha: \beta=1: 3$ ) and 3nitrophenylboronic acid (250 $\mathrm{mg}$ ) and was purified on silica gel chromatography (cyclohexane/EtOAc, from 90/10 to 50/50) to afford $28 \mathrm{mg}$ of the $\alpha$ anomer and 111 $\mathrm{mg}$ of the $\beta$ anomer as amorphous solids. Global yield: 59 $\%$ (o.29 mmol, $138 \mathrm{mg}$ ).

\section{1-(3'-Nitro)-phenyl-2,3,4,6-tetra-O-acetyl- $\alpha$-D- glucopyranoside. ${ }^{20}$}

${ }^{1} \mathrm{H}$ NMR (300 $\left.\mathrm{MHz}, \mathrm{CDCl}_{3}\right) \delta$ 8.04-7.86 (m, $2 \mathrm{H}$ ), 7.56-7.34 $(\mathrm{m}, 2 \mathrm{H}), 5.80(\mathrm{~d}, J=3.6 \mathrm{~Hz}, 1 \mathrm{H}), 5.68(\mathrm{app} \mathrm{t}, J=9.8 \mathrm{~Hz}, 1$ H), 5.15 (app t, $J=9.9 \mathrm{~Hz}, 1 \mathrm{H}), 5.06(\mathrm{dd}, J=10.3,3.6 \mathrm{~Hz}, 1$ $\mathrm{H}), 4.24(\mathrm{dd}, J=12.6,5.2 \mathrm{~Hz}, 1 \mathrm{H}), 4.14-3.97(\mathrm{~m}, 2 \mathrm{H}), 2.07$ $(\mathrm{s}, 3 \mathrm{H}), 2.05(\mathrm{~s}, 3 \mathrm{H}), 2.03(\mathrm{~s}, 3 \mathrm{H}), 2.02(\mathrm{~s}, 3 \mathrm{H})$.

${ }^{13} \mathrm{C}$ NMR (75 MHz, $\left.\mathrm{CDCl}_{3}\right) \delta$ 170.4, $170.0(2 \mathrm{C}), 169.4,156.5$, 149.2, 130.3, 122.9, 118.0, 111.9, 94.7, 70.2, 69.7, 68.6, 68.2, 61.6, 20.6, $20.5(3 \mathrm{C})$.

1-(3'-Nitro)-phenyl-2,3,4,6-tetra-O-acetyl- $\beta$-Dglucopyranoside. ${ }^{21}$

${ }^{1} \mathrm{H}$ NMR (300 $\mathrm{MHz}, \mathrm{CDCl}_{3}$ ) $\delta 7.95$ (ddd, $J=8.2,2.1,1.0 \mathrm{~Hz}$, $1 \mathrm{H}), 7.86$ (app t, $J=2.3 \mathrm{~Hz}, 1 \mathrm{H}), 7.47$ (app t, $J=8.2 \mathrm{~Hz}, 1$ H), 7.31 (ddd, $J=8.3,2.4,1.1 \mathrm{~Hz}, 1 \mathrm{H}), 5.40-5.25(\mathrm{~m}, 2 \mathrm{H})$, 5.24-5.06 (m, $2 \mathrm{H}), 4.22(\mathrm{~d}, J=4.2 \mathrm{~Hz}, 2 \mathrm{H}), 4.01-3.88(\mathrm{~m}, 1$ $\mathrm{H}), 2.09(\mathrm{~s}, 3 \mathrm{H}), 2.07(\mathrm{~s}, 3 \mathrm{H}), 2.06(\mathrm{~s}, 3 \mathrm{H}), 2.04(\mathrm{~s}, 3 \mathrm{H})$. ${ }^{13} \mathrm{C}$ NMR $\left(75 \mathrm{MHz} \mathrm{CDCl}_{3}\right) \delta$ 170.6, 170.1, 169.4, 169.2, 157.0, 149.1, 130.2, 123.7, 118.2, 111.3, 98.6, 72.5 (2 C), 71.0, 68.2, 62.0, $20.6(4 \mathrm{C})$.

\section{1-(3',4'-dichloro)-phenyl-2,3,4,6-tetra-O-acetyl-D- glucopyranoside 30 .}

The title compound was prepared according to the general procedure from commercially available 2,3,4,6-tetra-O-acetyl-D-glucopyranose (174 mg, $\alpha: \beta=1: 3$ ) and 3,4-dichlorophenylboronic acid $(288 \mathrm{mg})$ and was purified on silica gel chromatography (cyclohexane/EtOAc, from 9o/10 to 70/30) to afford $7 \mathrm{mg}$ of the $\alpha$ anomer and $53 \mathrm{mg}$ of the $\beta$ anomer as amorphous white solids. Global yield: $24 \%$ (o.12 mmol, $59 \mathrm{mg}$ ).

1-(3',5'-dichloro)-phenyl-2,3,4,6-tetra-O-acetyl- $\alpha$ - Dglucopyranoside.

${ }^{1} \mathrm{H}$ NMR (300 MHz, $\left.\mathrm{CDCl}_{3}\right) \delta 7.39(\mathrm{~d}, J=8.9 \mathrm{~Hz}, 1 \mathrm{H}), 7.28$ $(\mathrm{s}, 1 \mathrm{H}), 6.98(\mathrm{dd}, J=8.9,2.9 \mathrm{~Hz}, 1 \mathrm{H}), 5.7 \mathrm{o}(\mathrm{d}, J=3.3 \mathrm{~Hz}, 1$ 
H), 5.67 (app t, $J=9.8 \mathrm{~Hz}, 1 \mathrm{H}), 5.15($ app t, $J=9.7 \mathrm{~Hz}, 1$ H), $5.05(\mathrm{dd}, J=10.3,3.7 \mathrm{~Hz}, 1 \mathrm{H}), 4.26(\mathrm{dd}, J=12.9,5.6 \mathrm{~Hz}$, $1 \mathrm{H}), 4.17-3.97(\mathrm{~m}, 2 \mathrm{H}), 2.09-2.06(\mathrm{~m}, 12 \mathrm{H}) .{ }^{13} \mathrm{C}$ NMR $(75$ $\left.\mathrm{MHz} \mathrm{CDCl}_{3}\right) \delta 170.5,170.1$ (2 C), 169.5, 154.9, 133.2, 130.9, 126.7, 118.8, 116.3, 94.6, 70.3, 69.8, 68.4, 68.2, 61.6, 20.7, 20.6 (3 C). HRMS (ESI) calculated for $\mathrm{C}_{20} \mathrm{H}_{22} \mathrm{O}_{10} \mathrm{NaCl}_{2}$ $[\mathrm{M}+\mathrm{Na}]^{+}$515.0488, found 515.0483. $[\alpha]_{\mathrm{D}}{ }^{14}+186.7^{\circ}$ (c o.o8, $\mathrm{MeoH})$.

FT-IR $v_{\text {max }} / \mathrm{cm}^{-1}: 1739,1474,1383,1224,1033,932,826,776$, 687,608 .

1-(3',5'-dichloro)-phenyl-2,3,4,6-tetra-O-acetyl- $\beta$-Dglucopyranoside.

${ }^{1} \mathrm{H}$ NMR (300 $\mathrm{MHz}, \mathrm{CDCl}_{3}$ ) $\delta 7.34(\mathrm{~d}, J=8.8 \mathrm{~Hz}, 1 \mathrm{H}$ ), 7.12 $(\mathrm{d}, J=2.8 \mathrm{~Hz}, 1 \mathrm{H}), 6.84(\mathrm{dd}, J=8.9,2.8 \mathrm{~Hz}, 1 \mathrm{H}) 5.31-5.20$ (m, $2 \mathrm{H}), 5.12($ app t, $J=9.3 \mathrm{~Hz}, 1 \mathrm{H}), 5.03(\mathrm{~d}, J=7.1 \mathrm{~Hz}, 1$ $\mathrm{H}), 4.33-4.07$ (m, $2 \mathrm{H}$ ), 3.87 (ddd, $J=9.5,5.8,2.6 \mathrm{~Hz}, 1 \mathrm{H}$ ), 2.09 (s, $3 \mathrm{H}), 2.05(\mathrm{~s}, 3 \mathrm{H}), 2.04(\mathrm{~s}, 3 \mathrm{H}), 2.02(\mathrm{~s}, 3 \mathrm{H}) .{ }^{13} \mathrm{C}$ NMR (75 $\left.\mathrm{MHz}, \mathrm{CDCl}_{3}\right) \delta 170.5,170.1,169.3,169.2,155.5$, 133.0, 130.8, 126.9, 118.9, 117.0, 99.0, 72.5, 72.3, 71.0, 68.2, 62.0, 20.7, 20.6 (3 C). HRMS (ESI) calculated for $\mathrm{C}_{20} \mathrm{H}_{22} \mathrm{O}_{10} \mathrm{NaCl}_{2}[\mathrm{M}+\mathrm{Na}]^{+}$515.0488, found 515.0495. $[\alpha]_{\mathrm{D}}{ }^{14}-$ 22.2 ${ }^{\circ}$ (c 0.23, MeoH). FT-IR $v_{\text {max }} / \mathrm{cm}^{-1}:$ 1749, 1477, 1367, 1211, $913,873,825,711,600$.

\section{1-(2'-naphthyl)-2,3,4,6-tetra-O-acetyl-D- glucopyranoside $3 p$.}

The title compound was prepared according to the general procedure from commercially available 2,3,4,6-tetraO-acetyl-D-glucopyranose (174 mg, $\alpha: \beta=1: 3)$ and naphthalen-2-ylboronic acid $(258 \mathrm{mg})$ and was purified on silica gel chromatography (cyclohexane/EtOAc, from 90/10 to 70/30) to afford $64 \mathrm{mg}$ of the $\alpha$ anomer and 141 mg of the $\beta$ anomer as yellowish solids. Global yield: $86 \%$ (o.43 mmol, $203 \mathrm{mg}$ ).

1-(2'-naphthyl)-2,3,4,6-tetra-O-acetyl- $\alpha$-D-glucopyranoside.

${ }^{1} \mathrm{H}$ NMR (300 MHz, $\mathrm{CDCl}_{3}$ ) $\delta$ 7.83-7.75 (m, $3 \mathrm{H}$ ), 7.50-7.38 (m, $3 \mathrm{H}), 7 \cdot 31-7.28(\mathrm{~m}, 1 \mathrm{H}), 5.91(\mathrm{~d}, J=3.6 \mathrm{~Hz}, 1 \mathrm{H}), 5.79$ (app t, $J=9.8 \mathrm{~Hz}, 1 \mathrm{H}), 5.21$ (app t, $J=9.8 \mathrm{~Hz}, 1 \mathrm{H}), 5.13$ (dd, $J=10.2,3.6 \mathrm{~Hz}, 1 \mathrm{H}), 4.29(\mathrm{dd}, J=12.2,4.7 \mathrm{~Hz}, 1 \mathrm{H})$, 4.19 (ddd, $J=10.3,4.8,2.1 \mathrm{~Hz}, 1 \mathrm{H}$ ), 4.08 (dd, $J=12.2,2.1$ $\mathrm{Hz}, 1 \mathrm{H}), 2.09(\mathrm{~m}, 6 \mathrm{H}), 2.07(\mathrm{~s}, 3 \mathrm{H}), 2.00(\mathrm{~s}, 3 \mathrm{H}) \cdot{ }^{13} \mathrm{C}$ NMR $\left(75 \mathrm{MHz}, \mathrm{CDCl}_{3}\right) \delta 170.5,170.2$ (2 C), 169.6, 153.7, 134.2, 129.9, 129.7, 127.7, 127.1, 126.7, 124.7, 118.5, 110.9, 94.3, 70.5, 70.1, 68.4, 68.1, 61.6, 20.7, 20.6 (3 C). HRMS (ESI) calculated for $\mathrm{C}_{24} \mathrm{H}_{26} \mathrm{O}_{10} \mathrm{Na}[\mathrm{M}+\mathrm{Na}]^{+}$497.1424, found 497.1428. $[\alpha]_{\mathrm{D}}{ }^{14}+184.3^{\circ}\left(\right.$ c o.26, MeOH). FT-IR $\mathrm{v}_{\max } / \mathrm{cm}^{-1}$ : 1739, 1227, 1213, 118o, 1032, 973, 839, 751, 662, 609.

1-(2'-naphthyl)-2,3,4,6-tetra-O-acetyl- $\beta$-Dglucopyranoside. $^{22}$

${ }^{1} \mathrm{H}$ NMR (300 $\mathrm{MHz}, \mathrm{CDCl}_{3}$ ) $\delta$ 7.86-7.66 (m, $3 \mathrm{H}$ ), 7.52-7.35 $(\mathrm{m}, 2 \mathrm{H}), 7.34(\mathrm{~d}, J=2.5 \mathrm{~Hz}, 1 \mathrm{H}), 7.19$ (dd, $J=8.9,2.5 \mathrm{~Hz}, 1$ H), 5.43-5.28 (m, $2 \mathrm{H})$, 5.27-5.06 (m, $2 \mathrm{H}), 4.31(\mathrm{dd}, J=12.2$, $5.5 \mathrm{~Hz}, 1 \mathrm{H}), 4.21$ (dd, $J=12.3,2.5 \mathrm{~Hz}, 1 \mathrm{H}), 3.94$ (ddd, $J=$ 9.9, 5.6, $2.5 \mathrm{~Hz}, 1 \mathrm{H}), 2.08$ (m, 6H), 2.06 (s, $3 \mathrm{H}), 2.05$ (s, 3
H). $\left.{ }^{13} \mathrm{C} \mathrm{NMR} \mathrm{(75} \mathrm{MHz,} \mathrm{CDCl}_{3}\right) \delta 170.5,170.2,169.4,169.3$, 154.6, 134.1, 130.2, 129.7, 127.7, 127.1, 126.6, 124.7, 118.8, 111.5, $99.2,72.8,72.1,71.3,68.4,62.1,20.7,20.6$ (3 C).

\section{1-Phenyl-2,3,4,6-tetra-O-acetyl-D-mannopyranoside} 4 b.

The title compound was prepared according to the general procedure from 2,3,4,6-tetra-O-acetyl- $\alpha$-Dmannopyranose 1c (175 mg, $\alpha: \beta=9: 1)$ and phenylboronic acid (185 mg) and was purified on silica gel chromatography (cyclohexane/EtOAc, from 90/10 to 70/30) to afford $165 \mathrm{mg}$ of the $\alpha$ anomer and $27 \mathrm{mg}$ of the $\beta$ anomer as amorphous white solids. Global yield: 90 \% (0.45 mmol, $191 \mathrm{mg}$ ).

\section{1-Phenyl-2,3,4,6-tetra-O-acetyl- $\alpha$-D-mannopyranoside.}

${ }^{1} \mathrm{H}$ NMR (300 $\mathrm{MHz}, \mathrm{CDCl}_{3}$ ) $\delta$ 7.33-7.28 (m, $2 \mathrm{H}$ ), 7.11-7.04 $(\mathrm{m}, 3 \mathrm{H}), 5.57(\mathrm{dd}, J=10.0,3.5 \mathrm{~Hz}, 1 \mathrm{H}), 5.53(\mathrm{~d}, J=1.8 \mathrm{~Hz}$, $1 \mathrm{H}), 5.45$ (dd, $J=3.6,1.8 \mathrm{~Hz}, 1 \mathrm{H}), 5.37$ (app t, $J=10.2 \mathrm{~Hz}, 1$ H), 4.28 (dd, $J=12.4,5.5 \mathrm{~Hz}, 1 \mathrm{H}$ ), 4.19-4.02 (m, $2 \mathrm{H}), 2.20$ (s, $3 \mathrm{H}), 2.06(\mathrm{~s}, 3 \mathrm{H}), 2.04(\mathrm{~s}, 3 \mathrm{H}), 2.02(\mathrm{~s}, 3 \mathrm{H}) .{ }^{13} \mathrm{C}$ NMR $\left(75 \mathrm{MHz} \mathrm{CDCl}_{3}\right) \delta 170.5,169.9,169.9,169.7,155.6,129.6(2$ C), 123.0, 116.5 (2 C), 95.8, 69.4, 69.1, 68.9, 66.0, 62.1, 20.8, 20.7, 20.6 (2 C). HRMS (ESI) calculated for $\mathrm{C}_{20} \mathrm{H}_{24} \mathrm{O}_{10} \mathrm{Na}$ $[\mathrm{M}+\mathrm{Na}]^{+}$447.1267, found 447.1271. $[\alpha]_{\mathrm{D}}{ }^{14}+79.1^{\circ}(c \quad 0.22$, $\mathrm{MeOH})$. FT-IR $\mathrm{v}_{\max } / \mathrm{cm}^{-1}: 1743,1489,1366,1213,1124,1036$, 975, 915, 757, 692;

\section{1-Phenyl-2,3,4,6-tetra-O-acetyl- $\beta$-D-mannopyranoside.}

${ }^{1} \mathrm{H}$ NMR (3oo $\mathrm{MHz}, \mathrm{CDCl}_{3}$ ) $\delta$ 7.32-7.27 (m, $2 \mathrm{H}$ ), 7.17-6.78 $(\mathrm{m}, 3 \mathrm{H}), 5.69(\mathrm{~d}, J=3.3 \mathrm{~Hz}, 1 \mathrm{H}), 5.33$ (app t, $J=9.8 \mathrm{~Hz}, 1$ H), $5.24(\mathrm{~s}, 1 \mathrm{H}), 5.16$ (dd, $J=9.9,3.3 \mathrm{~Hz}, 1 \mathrm{H}), 4.35$ (dd, $J=$ 12.1, 6.2 Hz, $1 \mathrm{H}$ ), 4.22 (dd, $J=12.1,2.8 \mathrm{~Hz}, 1 \mathrm{H}$ ), 3.85 (ddd, $J$ = 9.4, 6.2, $2.8 \mathrm{~Hz}, 1 \mathrm{H}), 2.26(\mathrm{~s}, 3 \mathrm{H}), 2.10(\mathrm{~s}, 3 \mathrm{H}), 2.09(\mathrm{~s}, 3$ $\mathrm{H}), 2.04(\mathrm{~s}, 3 \mathrm{H}) .{ }^{13} \mathrm{C}$ NMR $\left(75 \mathrm{MHz}, \mathrm{CDCl}_{3}\right) \delta 170.5,170.3$, 169.9, 169.6, 156.6, 129.5 (2 C), 123.2, 116.8 (2 C), 96.9, 72.5, 70.9, 68.8, 66.1, 62.6, 20.8, 20.7 (2 C), 20.6. HRMS (ESI) calculated for $\mathrm{C}_{20} \mathrm{H}_{24} \mathrm{O}_{10} \mathrm{Na}[\mathrm{M}+\mathrm{Na}]^{+}$447.1267, found 447.1264. $[\alpha]_{\mathrm{D}}{ }^{14}-63.6^{\circ}$ (c o.44, MeOH). FT-IR $\mathrm{v}_{\max } / \mathrm{cm}^{-1}$ : 1735, 1492, 1369, 1214, 1053, 913, 844, 769, 694, 599.

\section{1-Phenyl-2,3,4,6-tetra-O-acetyl-D-galactopyranoside 4c.}

The title compound was prepared according to the general procedure from 2,3,4,6-tetra-O-acetyl- $\alpha$-Dgalactopyranose 1d (175 mg, $\alpha: \beta=2.3: 1)$ and phenylboronic acid ( $185 \mathrm{mg}$ ) and was purified on silica gel chromatography (cyclohexane/EtOAc, from 90/10 to $70 / 30)$ to afford $108 \mathrm{mg}$ of the $\alpha$ anomer and $57 \mathrm{mg}$ of the $\beta$ anomer as amorphous white solids. Global yield: $78 \%$ (o.39 mmol, $165 \mathrm{mg}$ ).

\section{1-Phenyl-2,3,4,6-tetra-O-acetyl- $\alpha$-D-galactopyranoside. ${ }^{23}$}

${ }^{1} \mathrm{H}$ NMR (300 $\mathrm{MHz} \mathrm{CDCl}_{3}$ ) $\delta$ 7.40-7.22 (m, $2 \mathrm{H}$ ), 7.09-7.04 $(\mathrm{m}, 3 \mathrm{H}), 5.79(\mathrm{~d}, J=3.6 \mathrm{~Hz}, 1 \mathrm{H}), 5.59(\mathrm{dd}, J=10.7,3.4 \mathrm{~Hz}$, $1 \mathrm{H}), 5.56-5.51(\mathrm{~m}, 1 \mathrm{H}), 5.30(\mathrm{dd}, J=10.7,3.6 \mathrm{~Hz}, 1 \mathrm{H}), 4.37$ (app t, $J=6.6 \mathrm{~Hz}, 1 \mathrm{H}), 4.17-4.04(\mathrm{~m}, 2 \mathrm{H}), 2.18(\mathrm{~s}, 3 \mathrm{H})$, 
$2.08(\mathrm{~s}, 3 \mathrm{H}), 2.04(\mathrm{~s}, 3 \mathrm{H}), 1.94(\mathrm{~s}, 3 \mathrm{H}) .{ }^{13} \mathrm{C}$ NMR $(75 \mathrm{MHz}$, $\left.\mathrm{CDCl}_{3}\right) \delta_{170.3}, 170.2,170.1,170.0,156.3,129.6$ (2 C), 123.0, 116.8 (2 C), 94.9, 67.9, 67.8, 67.5, 67.1, 61.5, 20.7, 20.6 (2 C), 20.5 .

\section{1-Phenyl-2,3,4,6-tetra-O-acetyl- $\beta$-D-galactopyranoside.$^{22}$}

${ }^{1} \mathrm{H}$ NMR (300 MHz, $\mathrm{CDCl}_{3}$ ) $\delta$ 7.36-7.26 (m, $2 \mathrm{H}$ ), 7.13-7.04 (m, $1 \mathrm{H}), 7.03-7.00(\mathrm{~m}, 2 \mathrm{H}), 5.50(\mathrm{dd}, J=10.5$, 8.0 Hz, $1 \mathrm{H})$, $5.45(\mathrm{~d}, J=3.2 \mathrm{~Hz}, 1 \mathrm{H}), 5.13(\mathrm{dd}, J=10.3,3.6 \mathrm{~Hz}, 1 \mathrm{H}), 5.06$ (d, $J=7.9 \mathrm{~Hz}, 1 \mathrm{H}), 4.34-3.96(\mathrm{~m}, 4 \mathrm{H}), 2.19(\mathrm{~s}, 3 \mathrm{H}), 2.07$ $(\mathrm{s}, 3 \mathrm{H}), 2.06(\mathrm{~S}, 3 \mathrm{H}), 2.02(\mathrm{~s}, 3 \mathrm{H}) .{ }^{13} \mathrm{C}$ NMR $(75 \mathrm{MHz}$, $\left.\mathrm{CDCl}_{3}\right) \delta_{170.3}, 170.2,170.1,169.3,156.9,129.5$ (2 C), 123.3, 116.9 (2 C), 99.7, 71.0, 70.8, 68.7, 66.9, 61.3, 20.7, 20.6 (3 C).

\section{1-Phenyl-2,3,5,6-di-O-isopropylidene- $\alpha$-D- mannofuranose $4 \mathrm{~d}^{24}$}

The title compound was prepared according to the general procedure from commercially available 2,3,5,6-di-Oisopropylidene- $\alpha$-D-mannofuranose $\quad(132 \mathrm{mg})$ and phenylboronic acid $(185 \mathrm{mg}$ ) and was purified on silica gel chromatography (cyclohexane/EtOAc, from 100/o to $80 / 20)$ to afford $128 \mathrm{mg}(76 \%)$ of $\alpha$ anomer only as a white amorphous solid.

${ }^{1} \mathrm{H}$ NMR (300 MHz, $\left.\mathrm{CDCl}_{3}\right) \delta$ 7.44-7.22 (m, $2 \mathrm{H}$ ), 7.06-7.01 $(\mathrm{m}, 3 \mathrm{H}), 5.66(\mathrm{~s}, 1 \mathrm{H}), 4.94(\mathrm{dd}, J=5.9,3.4 \mathrm{~Hz}, 1 \mathrm{H}), 4.89$ $(\mathrm{d}, J=5.9 \mathrm{~Hz}, 1 \mathrm{H}), 4.45$ (ddd, $J=7.9,6.2,4.2 \mathrm{~Hz}, 1 \mathrm{H}$ ), 4.21-4.06 (m, $2 \mathrm{H}), 4.01(\mathrm{dd}, J=8.7,4.2 \mathrm{~Hz}, 1 \mathrm{H}), 1.54(\mathrm{~s}, 3$ $\mathrm{H}), 1.45(\mathrm{~s}, 3 \mathrm{H}), 1.40(\mathrm{~s}, 6 \mathrm{H}) .{ }^{13} \mathrm{C} \mathrm{NMR}\left(75 \mathrm{MHz}, \mathrm{CDCl}_{3}\right) \delta$ $156.3,129.5$ (2 C), 122.2, 116.6 (2 C), 113.0, 109.4, 104.9, 85.5, 81.2, 79.6, 73.0, 66.9, 26.9, 26.0, 25.2, 24.6.

\section{1-Phenyl-2-deoxy-,3,4,6-tri-O-acetyl-D- glucopyranoside $4 \mathrm{e}$.}

The title compound was prepared according to the general procedure from 2-deoxy-3,4,6-tri-O-acetyl- $\alpha$ D-glucopyranose if ( $145 \mathrm{mg}, \alpha: \beta=8: 1)$ and phenylboronic acid $(185 \mathrm{mg})$ and was purified on silica gel chromatography (cyclohexane/EtOAc, from 90/10 to 50/50) to afford $78 \mathrm{mg}$ of the $\alpha$ anomer and $78 \mathrm{mg}$ of the $\beta$ anomer as white amorphous solids. Global yield: $89 \%$ ( $0.44 \mathrm{mmol}$, $163 \mathrm{mg}$ ).

\section{1-Phenyl-2-deoxy-,3,4,6-tri-O-acetyl- $\alpha$ - $D$ - glucopyranoside..$^{25}$}

${ }^{1} \mathrm{H}$ NMR (300 MHz, $\left.\mathrm{CDCl}_{3}\right) \delta$ 7.40-7.23 (m, $2 \mathrm{H}$ ), 7.19-6.91 $(\mathrm{m}, 3 \mathrm{H}), 5.70(\mathrm{~d}, J=3.5 \mathrm{~Hz}, 1 \mathrm{H}), 5.55$ (ddd, $J=11.5,9.4$, $5.4 \mathrm{~Hz}, 1 \mathrm{H}), 5.11$ (app t, $J=9.8 \mathrm{~Hz}, 1 \mathrm{H}), 4.31(\mathrm{dd}, J=12.1$, $4.7 \mathrm{~Hz}, 1 \mathrm{H}$ ), 4.08 (ddd, $J=10.1,4.7,2.2 \mathrm{~Hz}, 1 \mathrm{H}), 4.01$ (dd, $J$ = 12.2, $2.3 \mathrm{~Hz}, 1 \mathrm{H}), 2.49(\mathrm{dd}, J=13.1,5.4 \mathrm{~Hz}, 1 \mathrm{H}), 2.07(\mathrm{~s}, 3$ $\mathrm{H}), 2.06(\mathrm{~s}, 3 \mathrm{H}), 2.03(\mathrm{~s}, 3 \mathrm{H})$, 2.07-1.97 (m, $1 \mathrm{H}) .{ }^{13} \mathrm{C}$ NMR $\left(75 \mathrm{MHz}, \mathrm{CDCl}_{3}\right) \delta 170.6,170.2,169.9,156.1,129.5$ (2 C), 122.4, 116.4 (2 C), 95.2, 69.1, 68.9, 68.6, 62.1, 35.1, 21.0, 20.7 $(2 \mathrm{C})$.

1-Phenyl-2-deoxy-3,4,6-tri-O-acetyl- $\beta$-D-glucopyranoside.
${ }^{1} \mathrm{H}$ NMR (300 $\mathrm{MHz}, \mathrm{CDCl}_{3}$ ) $\delta$ 7.41-7.24 (m, $2 \mathrm{H}$ ), 7.09-7.01 $(\mathrm{m}, 3 \mathrm{H}), 5.22(\mathrm{dd}, J=9.6,2.2 \mathrm{~Hz}, 1 \mathrm{H}), 5.19-5.02(\mathrm{~m}, 2 \mathrm{H})$, $4.34(\mathrm{dd}, J=12.1,5.6 \mathrm{~Hz}, 1 \mathrm{H}), 4.17(\mathrm{dd}, J=12.2,2.6 \mathrm{~Hz}, 1$ H), 3.80 (ddd, $J=8.6,5.6,2.5 \mathrm{~Hz}, 1 \mathrm{H}$ ), 2.54 (ddd, $J=13.0$, 4.8, $2.2 \mathrm{~Hz}, 1 \mathrm{H}), 2.13^{-2.02}(\mathrm{~m}, 10 \mathrm{H}) .{ }^{13} \mathrm{C}$ NMR $(75 \mathrm{MHz}$, $\left.\mathrm{CDCl}_{3}\right) \delta 170.6,170.2,169.7,156.7,129.5$ (2 C), 122.8, 116.7 (2 C), 97.4, 72.2, 70.3, 68.9, 62.5, 35.9, 20.9, 20.7 (2 C). HRMS (ESI) calculated for $\mathrm{C}_{18} \mathrm{H}_{22} \mathrm{O}_{8} \mathrm{Na}[\mathrm{M}+\mathrm{Na}]^{+} 389.1212$, found 389.1205. $[\alpha]_{\mathrm{D}}{ }^{14}-30.3^{\circ}$ (c $\left.0.17, \mathrm{MeOH}\right)$. FT-IR $\mathrm{v}_{\max } / \mathrm{cm}^{-1}$ : 1735, 1494, 1364, 1228, 1115, 1050, 993, 914, 762, 695, 593.

\section{1-Phenyl-2,3,4,6-tetra-O-benzyl-D-glucopyranoside 4f. ${ }^{26}$}

The title compound was prepared according to the general procedure from commercially available 2,3,4,6-tetraO-benzyl- $\alpha$-D-glucopyranoside (270 $\mathrm{mg})$ and phenylboronic acid $(185 \mathrm{mg})$ and was purified on silica gel chromatography (cyclohexane/EtOAc, from 100/o to 90/10) to afford $252 \mathrm{mg}$ of an inseparable mixture of $\alpha$ and $\beta$ anomers as an amorphous white solid ( $82 \%, \alpha: \beta=1.2: 1$ in NMR, 1.4:1 in HPLC).

${ }^{1} \mathrm{H}$ NMR (300 MHz, $\mathrm{CDCl}_{3}$ ) $\delta$ 7.37-7.06 (m, $25 \mathrm{H} \alpha, 25 \mathrm{H} \beta$ ), $5.55(\mathrm{~d}, J=3.5 \mathrm{~Hz}, 1 \mathrm{H \alpha})$, 5.14-5.07 (m, $1 \mathrm{H \alpha}, 2 \mathrm{H} \beta)$, 5.04$4.84(\mathrm{~m}, 3 \mathrm{H \alpha}, 4 \mathrm{H} \beta$ ), 4.74 (app d, $J=12.0 \mathrm{~Hz}, 1 \mathrm{H \alpha}$ ), 4.68$4.55(\mathrm{~m}, 2 \mathrm{H \alpha}, 3 \mathrm{H} \beta), 4.47$ (app d, $J=12.0 \mathrm{~Hz}, 1 \mathrm{H} \alpha), 4.28$ (app t, $J=9.2 \mathrm{~Hz}, 1 \mathrm{H \alpha}$ ), 3.98-3.62 (m, $5 \mathrm{H \alpha}, 6 \mathrm{H} \beta) .{ }^{13} \mathrm{C}$ NMR $\left(75 \mathrm{MHz}, \mathrm{CDCl}_{3}\right) \delta 157.4,156.8,138.9,138.6,138.3$, 138.2 , 138.1, 137.9, 129.6, 128.5, 128.4, 128.3, 128.2, 128.0, $127.9,127.7,127.6,122.7,122.4$, 117.0, 116.9, 101.7, 95.5, 84.7, 82.1, 79.8, 77.8, 77.5, 75.8, 75.2, 75.1, 73.5, 73.3, 70.9, 68.9, 68.3. HRMS (ESI) calculated for $\mathrm{C}_{40} \mathrm{H}_{40} \mathrm{O}_{6} \mathrm{Na}[\mathrm{M}+\mathrm{Na}]^{+}$ 639.2723, found 639.2737.

\section{1-Phenyl-2-acetamido-3,4,6-tri-O-acetyl-2-deoxy-D- glucopyranose $4 \mathrm{~g}$.}

The title compound was prepared according to the general procedure from 2-acetamido-3,4,6-tri- $O$-acetyl-2deoxy-D-glucopyranose $\mathbf{l h}(172 \mathrm{mg}, \alpha: \beta=19: 1)$ and phenylboronic acid $(185 \mathrm{mg}$ ) and was purified on silica gel chromatography (cyclohexane/EtOAc, from 90/10 to 4o/6o) to afford $45 \mathrm{mg}$ of the $\alpha$ anomer and $12 \mathrm{mg}$ of the $\beta$ anomer as amorphous white solids. Global yield: $27 \%$ (o.135 mmol, $57 \mathrm{mg}$ ).

1-Phenyl-2-acetamido-3,4,6-tri-O-acetyl-2-deoxy- $\alpha$ - $D$ glucopyranose. ${ }^{27}$

${ }^{1} \mathrm{H}$ NMR (300 $\left.\mathrm{MHz}, \mathrm{CDCl}_{3}\right) \delta$ 7.28-7.20 (m, $2 \mathrm{H}$ ), 7.03-6.98 $(\mathrm{m}, 3 \mathrm{H}), 5.81(\mathrm{~d}, J=9.4 \mathrm{~Hz}, 1 \mathrm{H}), 5.50(\mathrm{~d}, J=3.6 \mathrm{~Hz}, 1 \mathrm{H})$, $5.37(\mathrm{dd}, J=10.8,9.4 \mathrm{~Hz}, 1 \mathrm{H}), 5.14($ app t, $J=9.7 \mathrm{~Hz}, 1 \mathrm{H})$, 4.45 (ddd, $J=10.8,9.4,3.6 \mathrm{~Hz}, 1 \mathrm{H}$ ), 4.14 (dd, $J=12.8$, 5.2 $\mathrm{Hz}, 1 \mathrm{H}), 4.00-3.96(\mathrm{~m}, 2 \mathrm{H}), 2.00(\mathrm{~s}, 3 \mathrm{H}), 1.97(\mathrm{~s}, 3 \mathrm{H})$, $1.95(\mathrm{~s}, 3 \mathrm{H}), 1.89(\mathrm{~s}, 3 \mathrm{H}) .{ }^{13} \mathrm{C}$ NMR $\left(75 \mathrm{MHz}, \mathrm{CDCl}_{3}\right) \delta$ 171.5, 170.6, 170.0, 169.3, 155.8, 129.7 (2 C), 123.1, 116.4 (2 C), 95.6, 71.1, 68.4, 67.9, 61.7, 51.9, 23.1, 20.7, 20.6 (2 C).

\section{1-Phenyl-2-acetamido-3,4,6-tri-O-acetyl-2-deoxy- $\beta$ - $D$ - glucopyranose. ${ }^{28}$}

${ }^{1} \mathrm{H}$ NMR (300 MHz, $\left.\mathrm{CDCl}_{3}\right) \delta$ 7.32-7.13 (m, $2 \mathrm{H}$ ), 7.04-6.79 $(\mathrm{m}, 3 \mathrm{H}), 5.61(\mathrm{~d}, J=8.7 \mathrm{~Hz}, 1 \mathrm{H}), 5.34(\mathrm{dd}, J=10.5,9.2 \mathrm{~Hz}$, 
$1 \mathrm{H}), 5.20(\mathrm{~d}, J=8.2 \mathrm{~Hz}, 1 \mathrm{H}), 5.07$ (app t, $J=9.5 \mathrm{~Hz}, 1 \mathrm{H})$, $4.22(\mathrm{dd}, J=12.2,5.4 \mathrm{~Hz}, 1 \mathrm{H}), 4.16-3.99$ (m, $2 \mathrm{H}), 3.90-3.70$ (m, 1 H), $2.00(\mathrm{~s}, 3 \mathrm{H}), 1.99(\mathrm{~s}, 3 \mathrm{H}), 1.97(\mathrm{~s}, 3 \mathrm{H}), 1.88(\mathrm{~s}, 3$ $\mathrm{H}) .{ }^{13} \mathrm{C} \mathrm{NMR}\left(75 \mathrm{MHz}^{\mathrm{C}} \mathrm{CDCl}_{3}\right) \delta 170.8,170.6,170.3,169.4$, 157.0, 129.5 (2 C), 123.2, 116.9 (2 C), 99.0, 72.1, 72.0, 68.6, 62.2, 54.8, 23.3, 20.7 (2 C), 20.6.

1-phenyl-2,3,6,2',3',4',6'-Hepta-O-acetylcellobiose 4i. The title compound was prepared according to the general procedure from $\mathbf{1 i}(320 \mathrm{mg}$ ) and phenylboronic acid (185 mg) and was purified on silica gel chromatography (cyclohexane/EtOAc, (50/50) to afford $274 \mathrm{mg}$ of an inseparable mixture of $\alpha$ and $\beta$ anomers as an amorphous white solid ( $77 \%, \alpha: \beta=1.5: 1$ in NMR, 1.2:1 in HPLC).

${ }^{1} \mathrm{H}$ NMR (300 MHz, $\left.\mathrm{CDCl}_{3}\right) \delta$ 7.26-7.20 (m, $2 \mathrm{H} \alpha, 2 \mathrm{H} \beta$ ), 7.02-6.96 (m, $3 \mathrm{H} \alpha, 1 \mathrm{H} \beta$ ), 6.91-6.88 (m, $2 \mathrm{H} \beta$ ), 5.63 (app t, $J=9.7 \mathrm{~Hz}, 1 \mathrm{H \alpha}), 5.57(\mathrm{~d}, J=3.5 \mathrm{~Hz}, 1 \mathrm{H} \alpha)$, 5.23-4.96 (m, 2 $\mathrm{H} \alpha, 5 \mathrm{H} \beta), 4.92-4.84(\mathrm{~m}, 2 \mathrm{H} \alpha, 1 \mathrm{H} \beta), 4.48-4.29$ (m, $3 \mathrm{H} \alpha$, $3 \mathrm{H} \beta)$, 4.11-3.94 (m, $3 \mathrm{H} \alpha, 2 \mathrm{H} \beta)$, 3.83-3.71 (m, $1 \mathrm{H} \alpha, 2 \mathrm{H} \beta)$, 3.62-3.59 (m, 1 H $\alpha, 1 \mathrm{H} \beta)$, 2.02-1.91 (m, $21 \mathrm{H \alpha}, 21 \mathrm{H} \beta) .{ }^{13} \mathrm{C}$ NMR (75 $\left.\mathrm{MHz} \mathrm{CDCl}_{3}\right) \delta 170.5,170.4,170.2,169.6,169.3$, 169.0, 156.8, 156.2, 129.6, 129.5, 123.3, 122.9, 116.9, 116.6, 100.8, 98.9, 94.1, 76.5, 76.4, 73.0, 72.9, 72.5, 72.0, 71.6, 71.4, 70.6, 69.5, 68.9, 67.8, 61.9, 61.6, 20.6, 20.5. HRMS (ESI) calculated for $\mathrm{C}_{32} \mathrm{H}_{40} \mathrm{O}_{18} \mathrm{Na}[\mathrm{M}+\mathrm{Na}]^{+}$735.2112, found 735.2112. $[\alpha]_{\mathrm{D}}^{14}+38.0^{\circ}$ (c o.40, DCM). FT-IR $\mathrm{v}_{\max } / \mathrm{cm}^{-1}$ : 1742, 1368, 1218, 1038, 908, 762, 693, 598.

\section{1-Phenyl-(4-O-(4-O-(2,3,4,6-tetra-O-acetyl- $\alpha-\mathrm{D}-$ glucopyranosyl)-2,3,6-tri-O-acetyl- $\alpha$-D- glucopyranosyl))-2,3,6-tri-O-acetyl-D-glucopyranose 4j.}

The title compound was prepared according to the general procedure from $\mathbf{1 j}$ (462 $\mathrm{mg}$ ) and phenylboronic acid $(185 \mathrm{mg}$ ) and was purified on silica gel chromatography (cyclohexane/EtOAc, (50/50) to afford $379 \mathrm{mg}$ of an inseparable mixture of $\alpha$ and $\beta$ anomers as a white solid $(76 \%, \alpha: \beta=1.6: 1$ in NMR, 1.6:1 in HPLC).

${ }^{1} \mathrm{H}$ NMR (300 MHz, $\left.\mathrm{CDCl}_{3}\right) \delta$ 7.30-7.18 (m, $2 \mathrm{H \alpha}, 2 \mathrm{H} \beta$ ), 7.06-6.98 (m, $3 \mathrm{H} \alpha, 1 \mathrm{H} \beta$ ), 6.93-6.9o (2 H $\beta$ ), 5.68 (app t, J $=9.5 \mathrm{~Hz}, 1 \mathrm{H \alpha}), 5.55(\mathrm{~d}, J=3.6 \mathrm{~Hz}, 1 \mathrm{H \alpha}), 5.37-5.22(\mathrm{~m}, 4$ $\mathrm{H} \alpha, 4 \mathrm{H} \beta$ ), 5.08-5.95 (m, $1 \mathrm{H} \alpha, 3 \mathrm{H} \beta)$, 4.87-4.75 (m, $2 \mathrm{H} \alpha, 1$ $\mathrm{H} \beta$ ), 4.71-4.67 (m, $1 \mathrm{H} \alpha, 1 \mathrm{H} \beta), 4.42-3.83(\mathrm{~m}, 11 \mathrm{H} \alpha, 12 \mathrm{H} \beta)$, 2.08-1.91 (m, 30 $\mathrm{H} \alpha, 30 \mathrm{H} \beta) .{ }^{13} \mathrm{C}$ NMR $\left(75 \mathrm{MHz}, \mathrm{CDCl}_{3}\right) \delta$ $170.7,170.6,170.5,170.4,170.3,170.0,169.7,169.6,169.4$, 156.7 , 156.2, 129.6, 123.3, 123.0, 117.0, 116.9, 98.5, 95.9, 95.7, 95.6, 94.3, 75.2, 73.9, 72.7, 72.5, 72.4, 72.2, 72.0, 71.7, 71.0, 70.5, 70.0, 69.4, 69.0, 68.6, 68.5, 68.o, 63.0, 62.6, 62.3, 61.4, 20.8, 20.7, 20.6, 20.5. HRMS (ESI) calculated for $\mathrm{C}_{44} \mathrm{H}_{56} \mathrm{O}_{26} \mathrm{Na}[\mathrm{M}+\mathrm{Na}]^{+}$1023.2958, found 1023.2950. $[\alpha]_{\mathrm{D}}{ }^{14}$ $+130.5^{\circ}$ (c $\left.0.48, \mathrm{MeOH}\right)$. FT-IR $\mathrm{v}_{\max } / \mathrm{cm}^{-1}: 1742,1368,1213$, 1029, 897, 761, 693, 6 oo.

(3aS,4R,6R,6aR)-4-phenoxy-2-phenyl-6-(2-phenyl1,3,2-dioxaborolan-4-yl)tetrahydrofuro[3,4d] $[1,3,2]$ dioxaborole 5 a.

Phenylboronic acid (245 mg, $2 \mathrm{mmol}$ ) and $\mathrm{MgSO}_{4}(2.0 \mathrm{~g}$, $16.6 \mathrm{mmol}$ ) were added to a suspension of $\alpha$-mannose $(180 \mathrm{mg}, 1.0 \mathrm{mmol})$ in dioxane $(20 \mathrm{~mL})$. The reaction me- dia was then stirred for three hours at room temperature, filtrated and the solvent was evaporated. The resulting diboronate was used without further purification. In a 100 $\mathrm{mL}$ round bottom flask, molecular sieves $(350 \mathrm{mg})$, $\mathrm{Cu}(\mathrm{OAc})_{2}{ }^{*} \mathrm{H}_{2} \mathrm{O}$ (60.0 mg, 0.30 mmol), pyridine (80.0 $\mu \mathrm{L}$, $1.0 \mathrm{mmol}$ ) and dichloroethane $(3.0 \mathrm{~mL})$ were introduced successively. The suspension was stirred 5 minutes and phenylboronic acid $(365 \mathrm{mg}, 3 \mathrm{mmol}$ ) was added, followed by the previous diboronate. This mixture was stirred under air atmosphere at room temperature for 24 hours. The media was diluted DCM and filtrated over celite. The solvent was removed by evaporation and the crude residue was purified by silica gel chromatographie (DCM) to afford $172 \mathrm{mg}(40 \%)$ of the title compound as a single $\alpha$ anomer.

${ }^{1} \mathrm{H}$ NMR (40o MHz, $\left.\mathrm{CDCl}_{3}\right) \delta$ 7.79-7.73 (m, $\left.4 \mathrm{H}\right), 7 \cdot 49-7.36$ (m, $2 \mathrm{H})$, 7.36-7.26 (m, $4 \mathrm{H})$, 7.24-7.16 (m, $2 \mathrm{H})$, 7.01-6.89 $(\mathrm{m}, 3 \mathrm{H}), 5.72(\mathrm{~s}, 1 \mathrm{H}), 5.24(\mathrm{dd}, J=6.2,3.9 \mathrm{~Hz}, 1 \mathrm{H}), 5.14$ (d, $J=6.1 \mathrm{~Hz}, 1 \mathrm{H}), 4.93($ app dt, $J=8.1,6.4 \mathrm{~Hz}, 1 \mathrm{H}), 4.4^{-}$ $4.31(\mathrm{~m}, 1 \mathrm{H}), 4.21(\mathrm{dd}, J=9.4,6.6 \mathrm{~Hz}, 1 \mathrm{H}) .{ }^{13} \mathrm{C}$ NMR $(100$ $\left.\mathrm{MHz}, \mathrm{CDCl}_{3}\right) \delta$ 156.0, 135.1 (2 C), 135.0 (2 C), 132.1, 131.6, 129.6 ( 2 C), 128.0 (2 C), 127.8 ( 2 C), 122.6, 116.7 (2 C), 105.2, 86.o, 82.3, 80.6, 74.3, 68.o. ${ }^{29}{ }^{11} \mathrm{~B}$ NMR (128 MHz, $\left.\mathrm{CDCl}_{3}\right) \delta$ 32.2. $[\alpha]_{D}{ }^{14}+106.4^{\circ}(c \quad 0.24, \mathrm{MeOH})$. FT-IR $\mathrm{v}_{\max } / \mathrm{cm}^{-1}: 1601$, 1369, 1297, 1223, 1097, 982, 754, 692, 634.

\section{1-Phenyl- $\alpha$-D-mannofuranose $6 a$.}

In a round bottom flask, 5a $(693 \mathrm{mg}, 1.62 \mathrm{mmol})$ was suspended in a mixture of $\mathrm{EtOH}(9.1 \mathrm{~mL})$ and water $(4.9$ $\mathrm{mL}$ ). $m$-CPBA ${ }^{30}$ (8oo $\mathrm{mg}, 3.24 \mathrm{mmol}$ ) was added and the resulting mixture was stirred at room temperature for 4 hours. The solvent was evaporated on a rotavapor and the crude residue was purified by silica gel chromatography (DCM/MeOH, from 100/o to 9o/10) to afford $334 \mathrm{mg}$ $(80 \%)$ of the titled compound as a colorless oil that solidified over time.

${ }^{1} \mathrm{H}$ NMR (300 MHz, $\left.\mathrm{CD}_{3} \mathrm{OD}\right) \delta$ 7.37-7.18 (m, $2 \mathrm{H}$ ), 7.04$6.96(\mathrm{~m}, 3 \mathrm{H}), 5.56(\mathrm{~d}, J=3.3 \mathrm{~Hz}, 1 \mathrm{H}), 4.39-4.33(\mathrm{~m}, 2 \mathrm{H})$, $4.11(\mathrm{dd}, J=8.1,3.1 \mathrm{~Hz}, 1 \mathrm{H}), 4.03-3.97(\mathrm{~m}, 1 \mathrm{H}), 3.78(\mathrm{dd}, J=$ 11.5 , 3.1 Hz, 1 H), 3.61 (dd, $J=11.5,6.0 \mathrm{~Hz}, 1 \mathrm{H}) .{ }^{13} \mathrm{C}$ NMR $(75$ $\left.\mathrm{MHz}, \mathrm{CD}_{3} \mathrm{OD}\right) \delta 158.8,130.4(2 \mathrm{C}), 123.0,117.7(2 \mathrm{C}), 107.7$, 81.6, 78.9, 72.6, 71.4, 64.7. HRMS (ESI) calculated for $\mathrm{C}_{12} \mathrm{H}_{16} \mathrm{O}_{6} \mathrm{Na}[\mathrm{M}+\mathrm{Na}]^{+}$279.0845, found 279.0850. $[\alpha]_{D}{ }^{14}$ $+171.4^{\circ}$ (c 0.32, MeOH). FT-IR $v_{\max } / \mathrm{cm}^{-1}: 3332,1599,1494$, 1222, 1007, 885, 823, 752, 690 .

\section{Synthesis of starting material $\mathbf{l b}, \mathbf{1 d}, \mathbf{1 f}, \mathbf{l h}, \mathbf{l i}$ and $\mathbf{1 j}$.}

\section{2,3,4,6-tetra- $\alpha$-O-acetyl-D-glucopyranose $1 b$.}

$\beta$-D-glucose pentacetate $(3.9 \mathrm{~g}$, $10.0 \mathrm{mmol})$ was introduced in THF $(50.0 \mathrm{~mL})$ and benzylamine $(1.2 \mathrm{~mL}$, 11.0 $\mathrm{mmol}$ ) was added. The resulting solution was stirred at room temperature for 18 hours. The media was diluted with $\mathrm{Et}_{2} \mathrm{O}$ and washed with a solution of $\mathrm{HCl}(1 \mathrm{M})$ and brine, dried over sodium sulfate and concentrated on a rotavapor. The crude residue was purified on silica gel chromatography (cyclohexahe/EtOAc, from 70/30 to $60 / 40)$ to afford the desired product as an inseparable mixture of two anomers. White foam, $2.9 \mathrm{~g}(82 \%, \alpha: \beta=$ 
3:1). NMR data were in good agreement with the literature. $^{31}$

${ }^{1} \mathrm{H}$ NMR (30o $\mathrm{MHz}, \mathrm{CDCl}_{3}$ ) $\delta$ ( $\alpha$ anomer) 5.54 (app t, $J=$ $9.8 \mathrm{~Hz}, 1 \mathrm{H}), 5.47($ app t, $J=3.7 \mathrm{~Hz}, 1 \mathrm{H}), 5.09$ (app t, $J=$ 9.8 Hz, $1 \mathrm{H}), 4.93-4.87$ (m, $1 \mathrm{H}), 4.29-4.22(\mathrm{~m}, 2 \mathrm{H}), 4.17^{-}$ $4.12(\mathrm{~m}, 1 \mathrm{H}), 3.52(\mathrm{~d}, J=3.9 \mathrm{~Hz}, 1 \mathrm{H}), 2.11-2.03(\mathrm{~m}, 12 \mathrm{H}),(\beta$ anomer) 5.26 (app t, $J=9.5 \mathrm{~Hz}, 1 \mathrm{H}), 5.13-5.06(\mathrm{~m}, 1 \mathrm{H})$, 4.93-4.87 (m, 1 H), 4.75 (app t, $J=8.3 \mathrm{~Hz}, 1 \mathrm{H}$ ), 4.29-4.22 $(\mathrm{m}, 1 \mathrm{H}), 4.17-4.12(\mathrm{~m}, 1 \mathrm{H}), 3.85(\mathrm{~d}, J=8.7 \mathrm{~Hz}, 1 \mathrm{H}), 3.77$ (ddd, $J=10.0,4.8,2.4 \mathrm{~Hz}, 1 \mathrm{H}), 2.11-2.03(\mathrm{~m}, 12 \mathrm{H}) .{ }^{13} \mathrm{C}$ NMR (75 MHz, $\mathrm{CDCl}_{3}$ ) $\delta$ 170.8, $170.7(\beta), 170.2,169.6,169.5$ ( $\beta)$, 95.5 $(\beta)$, 90.1 $(\alpha), 73.2(\beta)$, 72.3 $(\beta), 72.1(\beta), 71.1,69.9(\alpha)$, 68.5 $(\alpha), 68.5(\beta), 67.2(\alpha), 62.0(\alpha), 20.7,20.6$.

\section{2,3,4,6-Tetra-O-acetyl- $\alpha$-D-mannopyranose $1 c$.}

In a round bottom flask, D-mannose (3.06 g, $17 \mathrm{mmol}$ ) was dissolved in pyridine $(15.0 \mathrm{~mL}, 186 \mathrm{mmol})$. Then, acetic anhydride (16.0 mL, $170 \mathrm{mmol}$ ) was carefully added. The resulting mixture was stirred overnight at room temperature. The media was poured into water and the aqueous layer was extracted three times with EtOAc. The combined organic layer was then successively washed with $\mathrm{NaHCO}_{3}$ (sat), water and brine, dried over sodium sulfate and concentrated under reduced pressure to afford a brown foam. This intermediate was introduced in $\mathrm{Et}_{2} \mathrm{O}(85.0 \mathrm{~mL})$ and benzylamine $(2.05 \mathrm{~mL}, 18.7 \mathrm{mmol})$ was added. The resulting solution was stirred at room temperature for 5 hours. The media was then washed with $\mathrm{NH}_{4} \mathrm{Cl}$ (sat) and brine, dried over sodium sulfate and concentrated on a rotavapor. The crude residue was purified on silica gel chromatography (cyclohexahe/EtOAc, from $80 / 20$ to $50 / 50$ ) to afford the desired product as an inseparable mixture of two anomers. White foam, 4.1 g (70\%, $\alpha: \beta=9: 1)$. NMR data were in good agreement with the literature. ${ }^{32}$

${ }^{1} \mathrm{H}$ NMR (300 $\left.\mathrm{MHz}, \mathrm{CDCl}_{3}\right) \delta 5.41(\mathrm{dd}, J=10.1,3.2 \mathrm{~Hz}, 1 \mathrm{H})$, 5.32-5.22 (m, $3 \mathrm{H})$, 4.27-4.19 (m, $2 \mathrm{H}), 4.16-4.11(\mathrm{~m}, 1 \mathrm{H})$, $3.75(\mathrm{~d}, J=4.1 \mathrm{~Hz}, 1 \mathrm{H}), 2.15(\mathrm{~s}, 3 \mathrm{H}), 2.09$ (s, $3 \mathrm{H}), 2.04$ (s, 3 $\mathrm{H}), 1.99$ (s, $3 \mathrm{H}) .{ }^{13} \mathrm{C}$ NMR (75 $\left.\mathrm{MHz}, \mathrm{CDCl}_{3}\right) \delta 170.9,170.2$, 170.1, 169.8, 92.1, 70.1, 68.8, 68.5, 66.2, 62.6, 20.9 (2 C), 20.7 (2 C).

\section{2,3,4,6-Tetra-O-acetyl-D-galactopyranose $1 \mathrm{~d}$.}

In a round bottom flask, hydrazine acetate ( $0.26 \mathrm{~g}, \mathbf{2 . 8 2}$ mmol) was added to a solution of $\beta$-D-galactose pentaacetate (1.00 g, $2.56 \mathrm{mmol}$ ) in DMF (30 $\mathrm{mL})$. The resulting mixture was stirred at $55{ }^{\circ} \mathrm{C}$ for 2 hours. The media was allowed to cool down at room temperature and was diluted with EtOAc and a saturated solution of $\mathrm{NaHCO}_{3}$. The aqueous layer was extracted with EtOAc and the combined organic layer was washed with brine. The solvent was dried over $\mathrm{MgSO}_{4}$, filtrated and evaporated. The resulting oil was diluted with toluene and the volatiles were evaporated on a rotavapor. This procedure was performed until most of the DMF was eliminated. The crude residue was then purified by silica gel flash chromatography (cyclohexane/EtOAc, from 8o/20 to 40/6o) to afford the title compound as a mixture of two anomers. Amorphous white solid, $400 \mathrm{mg}$ (45\%, $\alpha: \beta=$
2.3:1). The NMR data were in good agreement with the literature. ${ }^{33}$

${ }^{1} \mathrm{H} \mathrm{NMR} \mathrm{(300} \mathrm{MHz}, \mathrm{CDCl}_{3}$ ) $\delta$ ( $\alpha$ anomer) 5.44 (app t, $J=$ $3.6 \mathrm{~Hz}, 1 \mathrm{H}), 5-41^{-} 5.40(\mathrm{~m}, 1 \mathrm{H}), 5.33-5.32(\mathrm{~m}, 1 \mathrm{H}), 5.07$ (dd, $J=10.8,3.5 \mathrm{~Hz}, 1 \mathrm{H}$ ), 4.41 (app t, $J=6.6 \mathrm{~Hz}, 1 \mathrm{H}), 4.10-$ $4.02(\mathrm{~m}, 2 \mathrm{H}), 3.93-3.90(\mathrm{~m}, 1 \mathrm{H}), 2.08(\mathrm{~s}, 3 \mathrm{H}), 2.03(\mathrm{~s}, 3 \mathrm{H})$, $1.99(\mathrm{~s}, 3 \mathrm{H}), 1.93(\mathrm{~s}, 3 \mathrm{H}),(\beta$ anomer $) 5.36(\mathrm{~d}, J=3.4 \mathrm{~Hz}, 1$ H),5.02-5.00 (m, $2 \mathrm{H}), 4.65$ (app t, $J=7.5 \mathrm{~Hz}, 1 \mathrm{H}), 4.14$ (d, $J=8.5 \mathrm{~Hz}, 1 \mathrm{H}), 4.10-4.02(\mathrm{~m}, 2 \mathrm{H}), 3.93-3.90(\mathrm{~m}, 1 \mathrm{H}), 2.10$ (s, $3 \mathrm{H}), 2.03(\mathrm{~s}, 3 \mathrm{H}), 1.99(\mathrm{~s}, 3 \mathrm{H}), 1.93(\mathrm{~s}, 3 \mathrm{H}) .{ }^{13} \mathrm{C}$ NMR $\left(75 \mathrm{MHz} \mathrm{CDCl}_{3}\right) \delta$ ( $\alpha$ anomer) 170.6, 170.5, 170.3, 170.2, 170.1, 90.6, 68.4, 68.2, 67.3, 66.1, 61.8, 20.8, 20.7, 20.6, 20.5, ( $\beta$ anomer) 170.9, 170.6, 170.2, 170.1, 95.9, 70.9 (2 C), 70.5, $67.2,61.5,20.8,20.7,20.6,20.5$.

2-Deoxy-3,4,6-tri-O-acetyl- $\alpha$-D-glucopyranose if.

In a round bottom flask and under inert atmosphere, $\mathrm{LiBr}$ (1.00 g, $11.52 \mathrm{mmol}$ ), amberlite IR120 (1.00 g) and water (1.2 $\mathrm{ml}$ ) were added to a solution of tri-O-acetyl-D-glucal (1.oo $\mathrm{g}, 3.67 \mathrm{mmol}$ ) in $\mathrm{MeCN}$ (30 mL). The solution was stirred at room temperature for $5 \mathrm{~h}$, filtered and neutralized with triethylamine. The volatiles were removed by evaporation on a rotavapor. The residue was dissolved in DCM and water. The organic phase was washed with an ice cold solution of $\mathrm{HCl}(\mathrm{lM})$ and $\mathrm{NaHCO}_{3}$ (sat), dried over $\mathrm{Na}_{2} \mathrm{SO}_{4}$, filtered and evaporated to dryness. Purification by flash column chromatography on silica gel (cyclohexane/EtOAc, 6o/40) afforded the desired product as a mixture of two anomers. White amorphous solid, $390 \mathrm{mg}$ $(37 \%, \alpha: \beta=8: 1)$. NMR data were in good agreement with the literature. ${ }^{34}$

${ }^{1} \mathrm{H}$ NMR (300 $\left.\mathrm{MHz}, \mathrm{CDCl}_{3}\right) \delta$ 5.43-5.34 (m, $2 \mathrm{H}$ ), 5.01 (app $\mathrm{t}, J=9.5 \mathrm{~Hz}, 1 \mathrm{H}), 4.30-4.19(\mathrm{~m}, 2 \mathrm{H}), 4.11-4.07(\mathrm{~m}, 1 \mathrm{H})$, 2.82-2.8o (m, 1 H), 2.28 (dd, $J=13.3,5.6 \mathrm{~Hz}, 1 \mathrm{H}), 2.09$ (s, 3 $\mathrm{H}), 2.04$ (s, $3 \mathrm{H}), 2.01$ (s, $3 \mathrm{H})$, 1.86-1.77 (m, $1 \mathrm{H}) .{ }^{1} \mathrm{H}$ NMR (300 $\mathrm{MHz} \mathrm{CDCl}_{3}$ ) $\delta 170.8,170.2,169.9,91.6,69.5,68.7$, 68.0, 62.5, 35.1, 20.9, 20.7(2 C).

\section{2-Acetamido-3,4,6-tri-O-acetyl-2-deoxy- $\alpha$-D- glucopyranose $\mathbf{t h}$.}

In a round bottom flask, glucosamine hydrochloride (2.0 $\mathrm{g}, 9.3 \mathrm{mmol})$ was introduced in pyridine $(26.0 \mathrm{~mL}, 322$ $\mathrm{mmol})$. Then, acetic anhydride (13.0 $\mathrm{mL}, 138 \mathrm{mmol}$ ) was carefully added. The resulting mixture was stirred overnight at room temperature. The media was poured into water and the aqueous layer was extracted three times with EtOAc. The combined organic layer was then successively washed with $\mathrm{NaHCO}_{3}$ (sat), water and brine, dried over sodium sulfate and concentrated under reduced pressure. This intermediate was dissolved in $\mathrm{Et}_{2} \mathrm{O}$ (40 mL) and $4.6 \mathrm{~mL}$ of a $2 \mathrm{M}$ solution of methylamine in $\mathrm{MeOH}$ (9.2 mmol) was then added. The resulting solution was stirred at room temperature for 4 hours. The media was then washed with $\mathrm{NH}_{4} \mathrm{Cl}$ (sat) and brine, dried over sodium sulfate and concentrated on a rotavapor. The crude residue was purified on silica gel chromatography (DCM/MeOH, from 100/o to 95/5) to afford the desired product as a mixture of two anomers. White amorphous 
solid, $2.4 \mathrm{~g}(73 \%, \alpha: \beta=19: 1)$. NMR data were in good agreement with the literature. ${ }^{35}$

${ }^{1} \mathrm{H}$ NMR (300 $\left.\mathrm{MHz}, \mathrm{CDCl}_{3}\right) \delta 6.00(\mathrm{~d}, J=9.3 \mathrm{~Hz}, 1 \mathrm{H}), 5.31-$ $5.22(\mathrm{~m}, 2 \mathrm{H}), 5.11$ (app t, $J=9.5 \mathrm{~Hz}, 1 \mathrm{H}), 4.67-4.66(\mathrm{~m}, 1$ $\mathrm{H})$, 4.30-4.07 (m, $4 \mathrm{H}), 2.07(\mathrm{~s}, 3 \mathrm{H}), 2.01(\mathrm{~s}, 4 \mathrm{H}), 2.01(\mathrm{~s}$, $\left.{ }_{4} \mathrm{H}\right), 1.95(\mathrm{~s}, 3 \mathrm{H}) .{ }^{13} \mathrm{C}$ NMR $\left(75 \mathrm{MHz}, \mathrm{CDCl}_{3}\right) \delta$ 171.4, 171.0, 170.6, 169.4, 91.5, 71.0, 68.3, 67.5, 62.1, 52.4, 23.1, 20.7 (2 C), 20.6 .

2,3,6,2',3',4',6'-Hepta-O-acetylcellobiose 1 i.

In a round bottom flask, $\alpha$-D-cellobiose octaacetate (1.36 $\mathrm{g}, 2.00 \mathrm{mmol}$ ) was dissolved in a mixture of THF (15.0 mL) and acetone $(2.0 \mathrm{~mL})$. To this solution was added benzylamine $(660 \mu \mathrm{L}, 6.0 \mathrm{mmol})$ and the resulting mixture was heated at $50{ }^{\circ} \mathrm{C}$ overnight. After cooling, the volatiles were evaporated and the residue was dissolved in DCM. The organic layer was successively washed with a solution of $\mathrm{HCl}(1 \mathrm{M}), \mathrm{NaHCO}_{3}$ (sat) and brine, dried over magnesium sulfate, filtrated and evaporated to dryness. Purification by flash column chromatography on silica gel (cyclohexane/EtOAc, 6o/40) afforded the desired product as a mixture of two anomers. White amorphous solid, 710 mg $(56 \%, \alpha: \beta=1.3: 1)$. NMR data were in good agreement with the literature. ${ }^{36}$

${ }^{1} \mathrm{H}$ NMR (3oo $\left.\mathrm{MHz}, \mathrm{CDCl}_{3}\right) \delta 5.48$ (app t, $J=9.7 \mathrm{~Hz}, 1 \mathrm{H} \alpha$ ), 5.34 (app t, $J=3.7 \mathrm{~Hz}, 1 \mathrm{H} \alpha$ ), 5.22-5.01 (m, $2 \mathrm{H} \alpha, 3 \mathrm{H} \beta$ ), 4.93-4.87 (m, $1 \mathrm{H} \alpha, 1 \mathrm{H} \beta$ ), 4.82-4.68 (m, $1 \mathrm{H} \alpha, 2 \mathrm{H} \beta$ ), 4.534.47 (m, $2 \mathrm{H} \alpha, 2 \mathrm{H} \beta$ ), 4.37-4.32 (m, $1 \mathrm{H} \alpha, 1 \mathrm{H} \beta$ ), 4.17-3.92 (m, $3 \mathrm{H} \alpha, 3 \mathrm{H} \beta$ ), 3.79-3.62 (m, $3 \mathrm{H} \alpha, 3 \mathrm{H} \beta$ ), 2.11-1.96 (m, 21 $\mathrm{H} \alpha, 21 \mathrm{H} \beta$ ). ${ }^{13} \mathrm{C}$ NMR (75 MHz, $\mathrm{CDCl}_{3}$ ) $\delta$ 170.6, 170.4, 170.3, 169.7, 169.3, 169.0, 100.8, 100.6, 95.2, 90.0, 76.5, 73.3, 73.0, $72.9,71.9,71.6,71.3,69.3,68.2,67.9,67.8,61.9,61.8,61.6$, 20.9, 20.7, 20.6, 20.5. HRMS (ESI) calculated for $\mathrm{C}_{26} \mathrm{H}_{36} \mathrm{O}_{18} \mathrm{Na}[\mathrm{M}+\mathrm{Na}]^{+}$654.2245, found 654.2251.

4-O-(4-O-(2,3,4,6-Tetra-O-acetyl- $\alpha-\mathrm{D}$ glucopyranosyl)-2,3,6-tri-O-acetyl- $\alpha$-Dglucopyranosyl)-2,3,6-tri-O-acetyl-D-glucopyranose 1j.

In a round bottom flask, sodium acetate (0.70 g, 8.30 $\mathrm{mmol}$ ) and maltotriose (3.00 g, $6.00 \mathrm{mmol}$ ) were added in acetic anhydride $(50 \mathrm{~mL})$. The reaction media was then refluxed under stirring for 2 hours. The mixture was allowed to cool down at room temperature and was diluted with DCM $(50 \mathrm{~mL})$. Water $(100 \mathrm{~mL})$ was carefully added and the media was stirred at room temperature for 10 minutes. The aqueous layer was extracted twice with DCM. The combined organic layers were washed twice with a solution of $\mathrm{NaHCO}_{3}$ (sat) and with brine, dried over magnesium sulfate and the solvent was evaporated to afford a yellow oil that was directly engaged in the next step. This crude product was dissolved in THF (20 mL) and benzylamine $(0.98 \mathrm{~mL}, 9.00 \mathrm{mmol})$ was added. The reaction mixture was stirred at room temperature overnight. Volatiles were evaporated, and the resulting residue was dissolved in DCM (50 $\mathrm{mL})$ and successively washed with a solution of $\mathrm{HCl}(1 \mathrm{M}), \mathrm{NaHCO}_{3}$ (sat) and brine. The organic layer was dried over magnesium sulfate, filtrated and the solvent was evaporated. Purification by silica gel chromatography (cyclohexane/EtOAc : $60 / 40$ ) afforded the desired compound as a mixture of two anomers. White amorphous solid, $3.05 \mathrm{~g}$ (55\%, $\alpha: \beta=$ 1.6:1). NMR data were in good agreement with the literature. $^{37}$

${ }^{1} \mathrm{H}$ NMR (30o $\left.\mathrm{MHz}, \mathrm{CDCl}_{3}\right) \delta 5.56$ (app t, $J=9.4 \mathrm{~Hz}, 1 \mathrm{H}$ ), 5.42-5.24 (m, $5 \mathrm{H} \alpha, 5 \mathrm{H} \beta$ ), 5.08-5.01 (m, $1 \mathrm{H \alpha}, 1 \mathrm{H} \beta$ ), 4.854.81 (m, $1 \mathrm{H} \alpha, 1 \mathrm{H} \beta$ ), 4.77-4.70 (m, $2 \mathrm{H} \alpha, 2 \mathrm{H} \beta$ ), 4.48-4.44 (m, $2 \mathrm{H} \alpha, 2 \mathrm{H} \beta$ ), 4.30-3.89 (m, $9 \mathrm{H} \alpha, 10 \mathrm{H} \beta)$, 3.78-3.74 (m, $1 \mathrm{H} \alpha, 1 \mathrm{H} \beta)$, 2.15-1.97 (m, 30 $\mathrm{H} \alpha, 30 \mathrm{H} \beta) .{ }^{13} \mathrm{C} \mathrm{NMR}(75$ $\left.\mathrm{MHz} \mathrm{CDCl}_{3}\right) \delta 170.7,170.6,170.5,170.4,170.2,169.9,169.8$, 169.7, 169.4, 95.8, 95.6, 94.9, 90.0, 74.7, 73.8, 73.7, 72.6, $72.5,72.4,72.3,71.8,71.7,71.6,70.4,70.1,69.4,69.0,68.9$, 68.5, 68.0, 67.7, 63.0, 62.4, 62.3, 61.4, 20.9, 20.8, 20.6, 20.5. HRMS (ESI) calculated for $\mathrm{C}_{38} \mathrm{H}_{52} \mathrm{O}_{26} \mathrm{Na} \quad[\mathrm{M}+\mathrm{Na}]^{+}$ 947.2645, found 947.2674 .

\section{ASSOCIATED CONTENT}

\section{Supporting Information}

The Supporting Information is available free of charge on the ACS Publications website.

Full optimization table, NMR spectra of all synthesized compounds and HPLC chromatograms.

\section{AUTHOR INFORMATION}

\section{Corresponding Author}

* samir.messaoudi@u-psud.fr, mouad.alami@u-psud.fr

\section{Author Contributions}

The manuscript was written through contributions of all authors. / All authors have given approval to the final version of the manuscript.

\section{ACKNOWLEDGMENTS}

Authors acknowledge support of this project by CNRS, University Paris-Sud, ANR (ANR-15-CE29- 0002), and by la Ligue Contre le Cancer through an Equipe Labellisée 2014 grant. We also thank the Algerian Ministry of Education and Research for a fellowship (PNE) to Sara Benmahdjoub. Our laboratory is a member of the Laboratory of Excellence LERMIT supported by a grant (ANR-10-LABX-33)

\section{REFERENCES}

(1) (a) Bililign, T. Griffith, B. R. Thorson, J. S. Structure, Activity, Synthesis and Biosynthesis of Aryl-C-glycosides. Nat. Prod. Prep. 2005, 22, 742; (b) Ovenden, S. P. B. Cobbe, M. Kissell, R. Birrell, G. W. Chavchich, M. Edstein, M. D. Phenolic Glycosides with Antimalarial Activity from Grevillea "Poorinda Queen”. J. Nat. Prod. 2011, 74, 74; (c) Perry, N. B. Brennan, N. J. Antimicrobial and Cytotoxic Phenolic Glycoside Esters from the New Zealand Tree Toronia Toru. J. Nat. Prod. 1997, 6o, 623; (d) Krenn, L. Presser, A. Pradhan, R. Bahr, B. Paper, D. H. Mayer, K. K. Kopp, B. Sulfemodin 8-O- $\beta$-D-Glucoside, a New Sulfated Anthraquinone Glycoside, and Antioxidant Phenolic Compounds from Rheum emodi. J. Nat. Prod. 2003, 66, 1107. 
(2) For a review, see: Jacobsson, M. Malmberg, J. Ellervik, U. Aromatic O-glycodylation. Carbohydr. Res. 2006, 341, 1266, and references therein.

(3) For recent review, see: Hain, J. Rollin, P. Klaffke, W. Lindhorst, T. K. Anomeric Modification of Carbohydrates Using the Mitsunobu Reaction. Beilstein J. Org. Chem. 2018, 14, 1619, and references therein.

(4) (a) Sharma, S. K. Corrales, G. Penade's, S. Single Step Stereoselective Synthesis of Unprotected 2,4-Dinitrophenyl Glycosides. Tetrahedron Lett. 1995, 36, 5627; (b) Koeners, H. J. de Kok, A. J. Romers, C. van Boom, J. H. The Use of the 2,4Dinitrophenyl Group in Sugar Chemistry Re-examined. Recl. Trav. Chim. Pays-Bas 1980, 99, 355; (c) Peterson, L. Jensen, K. J. A New, Efficient Glycosylation Method for Oligosaccharide Synthesis under Neutral Conditions: Preparation and Use of New DISAL Donors. J. Org. Chem. 2001, 66, 6268; (d) For the nonanomeric $O$-arylation, see: Henderson, A. S. Medina, S. Bower, J. F. Galan, M. C. Org. Lett. 2015, 17, 4846.

(5) (a) Tolnai, G. L. Nilsson, U. J. Olofsson, B. Efficient $O$ Functionalization of Carbohydrates with Electrophilic Reagents. Angew. Chem., Int. Ed. 2016, 55, 11226; (b) Lucchetti, N. Gilmour, R. Reengineering Chemical Glycosidation: Direct, Metal-Free Anomeric O-Arylation of Unactivated Carbohydrates. Chem. Eur. J. 2018, 24, 16266.

(6) (a) Ye, H. Xiao, C. Zhou, Q.-Q, Wang, P. G. Xiao, W.-J. Synthesis of Phenolyc Glycosides: Glycosylation of Sugar Lactols with Aryl Bromides via Dual Photoredox/Ni Catalysis. J. Org. Chem., 2018, 83, 13325.

(7) Bruneau, A. Brion, J.-D. Alami, M. Messaoudi, S. Stereoselective Copper-Catalyzed Chan-Lam-Evans $N$-Arylation of Glucosamines with Arylboronic Acids at Room Temperature. Chem. Commun. 2013. 49, 8359.

(8) A recent paper reported the use of Chan-Lam-Evans cross coupling for the $O$-arylation of the others hydroxyls groups of sugars but no anomeric $O$-arylation was reported: Dimakos, V. Garrett, E. G. Taylor, M. S. Site-Selective, Copper-Mediated OArylation of Carbohydrate Derivatives. J. Am. Chem. Soc. 2017, 139, 15515

(9) For a review related to the coupling of thiosugars, see: (a) Ibrahim, N. Alami, M. Messaoudi, S. Recent Advances in Transition Metal-Catalyzed Functionalization of 1-Thiosugars. Asian. J. Org. Chem. 2018; 7, 2026-2038; (b). Brachet, E.; Brion, J.D.; Messaoudi, S.; Alami, M. Stereoselective Palladium-Catalyzed Alkenylation and Alkynylation of Thioglycosides. Adv. Synth. Catal. 2013, 355, 2627. (c) Brachet, E.; Brion, J.-D.; Alami, M.; Messaoudi, S. Nickel-Catalyzed Arylation, Alkenylation, and Alkynylation of Unprotected Thioglycosides at Room Temperature. Chem. Eur. J. 2013, 19, 15276. (d) Chabrier, A.; Bruneau, A.; Benmahdjoub, S.; Benmerad, B.; Belaid, S.; Brion, J.-D.; Alami, M.; Messaoudi, S. Stereoretentive Copper-Catalyzed Directed Thioglycosylation of C(sp2)-H Bonds of Benzamides. Chem. Eur. J. 2016, 22, 15006. (e) AL-Shuaeeb, R. A. A.; Montoir, D.; Alami, M.; Messaoudi, S. J. Org. Chem. Synthesis of (1-2)-S-Linked Saccharides and S-Linked Glycoconjugates via a Palladium-G3XantPhos Precatalyst Catalysis. 2017, 82, 6720. (f) Probst, N.; Lartia, R.; Théry, O.; Alami, M.; Defrancq, E.; Messaoudi, S. Efficient Buchwald-Hartwig-Migita Cross-Coupling for DNA Thioglycoconjugation. Chem. Eur. J. 2018, 24, 1795. (g) Bruneau, A.; Roche, M.; Hamze, A.; Brion, J.-D.; Alami, M.; Messaoudi, S. Stereoretentive Palladium-Catalyzed Arylation, Alkenylation, and Alkynylation of 1-Thiosugars and Thiols Using
Aminobiphenyl Palladacycle Precatalyst at Room Temperature. Chem. Eur. J. 2015, 21, 8375.

(10) Axer A, Hermann S, Kehr G, Clases D, Karst U, FischerRiepe L, Roth J, Fobker M, Schäfers M, Gilmour R, Faust A. Harnessing the Maltodextrin Transport Mechanism for Targeted Bacterial Imaging: Structural Requirements for Improved in vivo Stability in Tracer Design. ChemMedChem. 2018, 13, 241

(11) Manhas, S. Taylor, M. S. Boronic Acids as Phase-Transfer Reagents for Fischer Glycosidations in Low-Polarity Solvents. J. Org. Chem. 2017, 82, 11406.

(12) (a) Vantourout, J. C.; Miras, H. N.; Isidro-Llobet, A.; Sproules, S.; Watson, A. J. B. Spectroscopic studies of the Chan-Lam amination: A mechanism inspired solution to boronic ester reactivity J. Am. Chem. Soc., 2009, 131, 5044; (b) King, A. E. Brunold, T. C. Stahl, S. S. Mechanistic Study of Copper-Catalyzed Aerobic Oxidative Coupling of Arylboronic Esters and Methanol: Insights into an Organometallic Oxidase Reaction. Am. Chem. Soc., 2009, 131, 5044; (c) King, A. E. Ryland, B. L. Brunold, T. C. Stahl, S. S. Kinetic and Spectroscopic Studies of Aerobic Copper(II)-Catalyzed Methoxylation of Arylboronic Esters and Insights into Aryl Transmetalation to Copper(II), Organometallics 2012, 31, 7948.

(13) M. Matsui, M. Okada, Chem. Pharm. Bull. 1972, 20, 1033.

(14) E. J. Cocinero, D. P. Gamblin, B. G. Davis, J. P. Simons, J. Am. Chem Soc. 2009, 131, 11117.

(15) C. J. Capicciotti, J. D. R. Kurach, T. R. Turner, R. S. Mancini, J. P. Acker, R. N. Ben, Sci. Rep. 2015, 5, article 9692.

(16) C. J. Capicciotti, R. S. Mancini, T. R. Turner, T. Koyama, M. G. Alteen, M. Doshi, T. Inada, J. P. Acker, R. N. Ben, ACS Omega 2016 $1,656-662$.

(17) C. Socolsky, M. L. Fascio, N. B. D'Accorso, A. Salvatore, E. Willink, Y. Asakawa, A. Bardon, J. Chem. Ecol. 2008, 34, 539-548.

(18) D. Schwizer, H. Gäthje, S. Kelm, M. Porro, O. Schwardt, B. Ernst, Bioorg. Med. Chem. 2006, 14, 4944-4957.

(19) S. Al Bittar, N. Mora, M. Loonis, O. Dangles, Tetrahedron 2016, 72, 4294-4302.

(20) Y. Li, H. Mo, G. Lian, B. Yu, Carbohydrate Research 2012, 363, 14-22.

(21) A. Temeriusz, T. Gubica, P. Rogowska, K. Paradowska, M. K. Cyranski, Carbohydrate Research 2005, 340, 1175-1184.

(22) I. J. Talisman, V. Kumar, J. R. Deschamps, M. frisch, S. V. Malhotra, Carbohydrate Research 2011, 346, 2337-2341.

(23) G. St-Pierre, L. Dafik, E. Klegraf, S. Hanessian, Synthesis 2016 , 48, 3575-3588.

(24 G. L. Tolnai, U. J. Nilsson, B. Olofsson, Angew. Chem. Int. Ed. 2016, 55, 11226-11230.

(25 G. Yang, Q. Wang, X. Luo, J. Zhang, J. Tang, Glycoconj. J. 2012, $29,453-456$

(26) H. Ye, C. Xiao, Q-Q. Zhou, P. G. Wang, W-J. Xiao, J. Org. Chem. 2018, 83, 13325-13334 ; R. R. France, N. V. Rees, J. D. Wadhawan, A. J. Fairbanks, R. G. Compton, Org. Biomol. Chem. 2004, 2, 2188-2194; R. Dyapa, L. T. Dockery, M. A. Walcazak, Org. Biomol. Chem. 2017, 15, 51-55.

(27) D. Frem, D. Urban, S. Norsikian, J-M. Beau, Eur. J. Org. Chem. 2017, 5094-5101.

(28) T. A. Chupakhina, V. O. Kur'yanov, V. Ya. Chirva, R. Ya. Grigorash, S. A. Kotlyar, G. L. Kamalov, Russ. J. Bioorganic Chem. 2004, 30, 301-303.

(29) Carbons in the alpha position of boron atoms are not observed. (30) $75 \%$ of actual $m$-CPBA, in a mixture with remaining metachlorobenzoic acid and water.

(31) T. B. Cai, D. Lu, X. Tang, Y. Zhang, M. Landerholm, P. G. Wang, J. Org. Chem. 2005, 70, 3518-3524.

(32) H. G. Sudibya, J. Ma, X. Dong, S. Ng, L-J. Li, X-W. Liu, P. Cheng, Angew. Chem., Int. Ed. 2009, 48, 2723-2726. 
(33) S. M. Andersen, M. Heuckendorff, H. H. Jensen, Org. Lett. 2015, 17, 944-947.

(34) C. Bucher, R. Gilmour, Angew. Chem., Int. Ed. 2010, 49, 87248728 .

(35) B. K. Gorityala, Z. Lu, M. L. Leow, J. Ma, X-W. Liu, J. Am. Chem. Soc. 2012, 134, 15229-15232.

(36) R. Khan, P. A. Konowicz, L. Gardossi, M. Matulova, S. De Gennaro, Aust. J. Chem. 1996, 49, 293-298.

(37) R. Wang, J-Z. Chen, X-A. Zheng, R. Kong, S-S. Gong, Q. Sun, Carbohydrate Research 2018, 455, 114-118. 